\title{
Quark mass dependence of on-shell and half off-shell scattering amplitudes from Bethe-Salpeter wave function inside the interaction range
}

\author{
Yusuke Namekawa \\ Faculty of Pure and Applied Sciences, University of Tsukuba, Tsukuba, Ibaraki 305-8571, Japan \\ Takeshi Yamazaki \\ Faculty of Pure and Applied Sciences, University of Tsukuba, Tsukuba, Ibaraki 305-8571, Japan \\ and Center for Computational Sciences, University of Tsukuba, Tsukuba, Ibaraki 305-8577, Japan
}

(Received 2 April 2019; published 24 June 2019)

\begin{abstract}
We evaluate scattering amplitudes at on-shell and half off-shell for $I=2 \mathrm{~S}$-wave two-pion system using the Bethe-Salpeter wave function inside the interaction range in the quenched QCD. The scattering length and effective range are extracted from these scattering amplitudes. Quark mass dependence of them is investigated with the pion mass ranged in $0.52-0.86 \mathrm{GeV}$. We examine consistency between a result by the conventional finite volume method and our estimate, as well as the phenomenological value.
\end{abstract}

DOI: 10.1103/PhysRevD.99.114508

\section{INTRODUCTION}

Lattice QCD has contributed to quantitative understanding of hadrons from the first principle of the strong interaction. Hadron scattering represented by the scattering length $a_{0}$, the effective range $r_{\text {eff }}$, and the scattering phase shift $\delta(k)$ itself, can be obtained by lattice QCD. The recent works are summarized in Refs. [1,2].

The scattering information is extracted by the finite volume method and the extensions, formulated by M. Lüscher [3,4]. An analytic function describes the relation between two-hadron energies in a finite volume and the scattering phase shift in the infinite volume. This relation is derived by use of the Bethe-Salpeter (BS) wave function outside the interaction range $R$ of two hadrons in quantum field theory [5,6]. A method to define a potential between hadrons from the BS wave function is also proposed [7].

An associated issue between the on-shell scattering amplitude and the BS wave function inside $R$ is argued in the infinite volume $[5,6,8]$. The $S$-wave two-pion scattering amplitude is defined by the Lehmann-SymanzikZimmermann (LSZ) reduction formula under an assumption that inelastic scattering effects are negligible. In the center of mass frame, the half off-shell amplitude $H(p ; k)$ is related to the pion four-point function,

Published by the American Physical Society under the terms of the Creative Commons Attribution 4.0 International license. Further distribution of this work must maintain attribution to the author(s) and the published article's title, journal citation, and DOI. Funded by SCOAP.

$$
\begin{aligned}
e^{-i \vec{q} \cdot \vec{x}} & \frac{-i \sqrt{Z}}{-\vec{q}^{2}+m_{\pi}^{2}-i \epsilon} \frac{4 E_{p} E_{k}}{E_{p}+E_{k}} H(p ; k) \\
= & \int d^{4} z d^{4} y_{1} d^{4} y_{2} K(\vec{p}, \vec{z}) K\left(-\vec{k}_{1}, \vec{y}_{1}\right) K\left(-\vec{k}_{2}, \vec{y}_{2}\right) \\
& \times\left\langle 0\left|\pi_{1}(\vec{z}) \pi_{2}(\vec{x}) \pi_{1}\left(\vec{y}_{1}\right) \pi_{2}\left(\vec{y}_{2}\right)\right| 0\right\rangle,
\end{aligned}
$$

where the vectors are four dimensional. The two-pion energy is $E_{k}=2 \sqrt{m_{\pi}^{2}+k^{2}}$, where $k=|\mathbf{k}|$ is a magnitude of three-dimensional momentum. The kernel is $K(\vec{p}, \vec{z})=i e^{i \vec{p} \cdot \vec{z}}\left(-\vec{p}^{2}+m^{2}\right) / \sqrt{Z}$ with $Z$ being the renormalization factor of the pion operator $\pi_{i}$. The three momenta $\vec{p}, \overrightarrow{k_{1}}$, and $\overrightarrow{k_{2}}$ are on-shell. Contrarily, $\vec{q}$ is in general off-shell. The on-shell scattering amplitude $H(k ; k)$ is associated with the scattering phase shift $\delta(k)$ through

$$
H(k ; k)=\frac{4 \pi}{k} e^{i \delta(k)} \sin \delta(k) .
$$

Some ratio is needed to cancel out $e^{i \delta(k)}$, which can not be measured directly on the lattice. We divide $H(k ; k)$ by the BS wave function at a reference point to remove $e^{i \delta(k)}$. The details are explained in Sec. IV.

Based on this issue, we accomplished a simulation to obtain scattering amplitudes from the BS wave function inside $R$ on a finite volume lattice [9]. The simulation utilized the isospin $I=2 \mathrm{~S}$-wave two pions in the quenched QCD with the lattice spacing of $a^{-1}=$ $1.207 \mathrm{GeV}$ at the pion mass $m_{\pi}=0.86 \mathrm{GeV}$. Using the on-shell amplitude, we numerically confirmed agreement 
between a result by the finite volume method and that by our approach. We also presented that lattice QCD can successfully give the half off-shell scattering amplitude. Although the half off-shell scattering amplitude is not an observable in experiments, it gives constraints on the parameters of effective theories and models of the hadron interaction by comparing the half off-shell amplitude from the lattice QCD to those of effective theories and models. An example of the calculation in the continuum theory is presented in Ref. [10]. Lattice QCD results of the half offshell scattering amplitude can be useful as supplementary data to effective theories and models.

In this paper, we extend our previous simulation [9] to investigate the scattering amplitudes with several pion masses $m_{\pi}=0.52-0.86 \mathrm{GeV}$ at the same lattice spacing. The results are extrapolated to the physical point to examine consistency among the previous result by the conventional finite volume method, the phenomenological estimate, and our result.

This paper is organized as follows. Section II is devoted to the formulation in the lattice theory. In Sec. III, details of our simulation set up are presented. Section IV explains our results at each $m_{\pi}$, as well as those at the physical point. Comparison with the previous lattice QCD results and the phenomenological values is also given in this section. Section V summarizes this paper. The Appendixes contain the operator dependence of the scattering amplitude, the formulation of the scattering amplitude using the BS wave function in the momentum space, and a discussion on the time dependence of the scattering amplitude.

\section{FORMULATION ON THE LATTICE}

Formulation of the scattering amplitude is explained. We use the same notation in our previous work [9], following Refs. $[5,6,8]$. We restrict ourselves to the S-wave scattering of $I=2$ two pions in the center of mass frame, assuming inelastic scattering effects are negligible. The two-pion BS wave function on the lattice $\phi(\mathbf{x} ; k)$ is obtained by

$$
\phi(\mathbf{x} ; k)=\left\langle 0|\Phi(\mathbf{x}, 0)| \pi^{+} \pi^{+}, E_{k}\right\rangle,
$$

where $\left|\pi^{+} \pi^{+}, E_{k}\right\rangle$ is a ground state of two pions, and $E_{k}=$ $2 \sqrt{m_{\pi}^{2}+k^{2}}$ is the two-pion energy. $\Phi(\mathbf{x}, t)$ is an operator of two pions,

$$
\Phi(\mathbf{x}, t)=\sum_{\mathbf{r}} \pi^{+}\left(R_{A_{1}^{+}}[\mathbf{x}]+\mathbf{r}, t\right) \pi^{+}(\mathbf{r}, t)
$$

with $A_{1}^{+}$projection $R_{A_{1}^{+}}[\mathbf{x}] . R_{A_{1}^{+}}[\mathbf{x}]$ is performed to obtain the $\mathrm{S}$-wave scattering state with an assumption that higher angular momentum contributions of $l \geq 4$ scattering are negligible. We remark that the tininess of the $l=4$ contribution in the $I=2$ two-pion system has been explicitly confirmed at $m_{\pi}=396 \mathrm{MeV}$ using the finite volume method [11]. The pion interpolating operator is defined by

$$
\pi^{+}(\mathbf{x}, t)=\bar{d}(\mathbf{x}, t) \gamma_{5} u(\mathbf{x}, t) .
$$

$\phi(\mathbf{x} ; k)$ is related to a pion four-point function $C_{\pi \pi}(\mathbf{x}, t)$ such that

$$
\begin{aligned}
C_{\pi \pi}(\mathbf{x}, t) & =\left\langle 0\left|\Phi\left(\mathbf{x}, t_{\text {sink }}\right) \Omega^{\dagger}\left(t_{\text {src }}\right)\right| 0\right\rangle \\
& =C_{k} \phi(\mathbf{x} ; k) e^{-E_{k} t}+\cdots,
\end{aligned}
$$

where $\Omega\left(t_{\mathrm{scc}}\right)$ is an operator of a two-pion at the source time slice $t_{\mathrm{src}}$, and $t=\left|t_{\text {sink }}-t_{\mathrm{src}}\right|$. The dot term corresponds to excited state contributions. $C_{k}$ is an overall constant.

An essential quantity to calculate the scattering amplitude from $\phi(\mathbf{x} ; k)$ is the reduced BS wave function $h(\mathbf{x} ; k)$ $[6,8]$. It is defined through $\phi(\mathbf{x} ; k)$ as

$$
h(\mathbf{x} ; k)=\left(\Delta+k^{2}\right) \phi(\mathbf{x} ; k),
$$

where

$$
\Delta f(\mathbf{x})=\sum_{i=1}^{3}(f(\mathbf{x}+\hat{i})+f(\mathbf{x}-\hat{i})-2 f(\mathbf{x})) .
$$

$h(\mathbf{x} ; k)$ possesses an important property that $h(\mathbf{x} ; k)$ equals to zero outside the interaction range of two pions $R$, except for the exponential tail,

$$
h(\mathbf{x} ; k)=0 \text { for } x>R .
$$

$h(\mathbf{x} ; k)$ defines the half off-shell amplitude on the lattice $H_{L}(p ; k)$ with an off-shell momentum $p$,

$$
H_{L}(p ; k)=-\sum_{\mathbf{x} \in L^{3}} C_{k} h(\mathbf{x} ; k) j_{0}(p x),
$$

where $j_{0}(p x)$ is the spherical Bessel function. If $R$ is less than half of the lattice extent $L, R<L / 2$, and the exponential tail is negligible, then the range of the summation can be changed from $L$ to $\infty$ due to Eq. (10), as discussed in Ref. [9]. $H_{L}(p ; k)$ becomes the half off-shell amplitude in the infinite volume $H(p ; k)$ in Eq. (1) as

$$
H(p ; k)=\frac{H_{L}(p ; k)}{C_{00}},
$$

except for an overall finite volume correction, $C_{00}=$ $C_{k} / F(k, L) . F(k, L)$ is a finite volume correction of the two-pion state, called the Lellouch and Lüscher factor [12]. $F(k, L)$ is defined by $\left|\pi^{+} \pi^{+}\right| \pi^{+} \pi^{+}, E_{k}>_{L=\infty}=$ $F(k, L) \mid \pi^{+} \pi^{+}, E_{k}>_{L}$. The finite volume correction of $H(p ; k)$ is removed by normalizing with the on-shell value $H(k ; k)$, presented in Sec. IV. 
TABLE I. Simulation parameters.

\begin{tabular}{lccc}
\hline \hline Lattice size & $\kappa_{\text {val }}$ & $N_{\text {src }}$ & $N_{\text {config }}$ \\
\hline $24^{3} \times 96$ & $0.1340,0.1358,0.1369$ & 24 & 200 \\
\hline \hline
\end{tabular}

As explained above, $R<L / 2$ with a negligible exponential tail of $h(\mathbf{x} ; k)$ is the sufficient condition for the scattering amplitude calculation on the lattice. We numerically confirm this condition is satisfied in the later section.

\section{SETUP}

Our simulation setup is presented. We perform a quenched QCD simulation following Refs. [6,13]. We generate 200 gauge configurations on a $24^{3} \times 96$ lattice by the Hybrid Monte Carlo algorithm, stored at every 100 trajectories. Our gauge action is the Iwasaki type [14]. The lattice spacing is $a^{-1}=1.207 \mathrm{GeV}$; the bare coupling is $\beta=2.334$. Our valence quark action is a Clover type [15]. The Clover coefficient is mean field improved, $C_{\mathrm{SW}}=1.398$ [13]. Our pion masses are in range of $m_{\pi}=0.86-0.52 \mathrm{GeV}$ with the valence quark hopping parameters of $\kappa_{\mathrm{val}}=0.1340-0.1369$. Our simulation parameters are collected in Table I.

We also generate gauge configurations on the $24^{3} \times 64$ lattice with the same setup to investigate the source operator dependence of the scattering amplitude on the lattice. Details of the investigation are explained in Appendix A.

The two-pion four-point function $C_{\pi \pi}(\mathbf{x}, t)$ in Eq. (6) is calculated using complex random $Z 2$ sources to avoid Fierz contamination,

$$
\Omega(t)=\frac{1}{N_{r}\left(N_{r}-1\right)} \sum_{\substack{i, j=1 \\ i \neq j}}^{N_{r}} \pi^{+}\left(t, \eta_{i}\right) \pi^{+}\left(t, \eta_{j}\right),
$$

where $\Omega(t)$ is the source operator in Eq. (6). $\pi^{+}(t, \eta)$ is defined by

$\pi^{+}(t, \eta)=\left[\sum_{\mathbf{x}_{1}} \bar{d}\left(\mathbf{x}_{1}, t\right) \eta^{\dagger}\left(\mathbf{x}_{1}\right)\right] \gamma_{5}\left[\sum_{\mathbf{x}_{2}} u\left(\mathbf{x}_{2}, t\right) \eta\left(\mathbf{x}_{2}\right)\right]$.

$N_{r}$ is the number of the random sources $\eta_{i}(\mathbf{x})$, satisfying the following condition:

$$
\frac{1}{N_{r}} \sum_{i=1}^{N_{r}} \eta_{i}^{\dagger}(\mathbf{x}) \eta_{i}(\mathbf{y}) \underset{N_{r} \rightarrow \infty}{\longrightarrow} \delta(\mathbf{x}-\mathbf{y}) .
$$

$N_{r}=4$ is employed in our simulation. The source resides at a time $t_{\text {src }}$ in Eq. (6) and all spatial points, as well as all colors and spins. The latter reduces the simulation cost [16]. In our
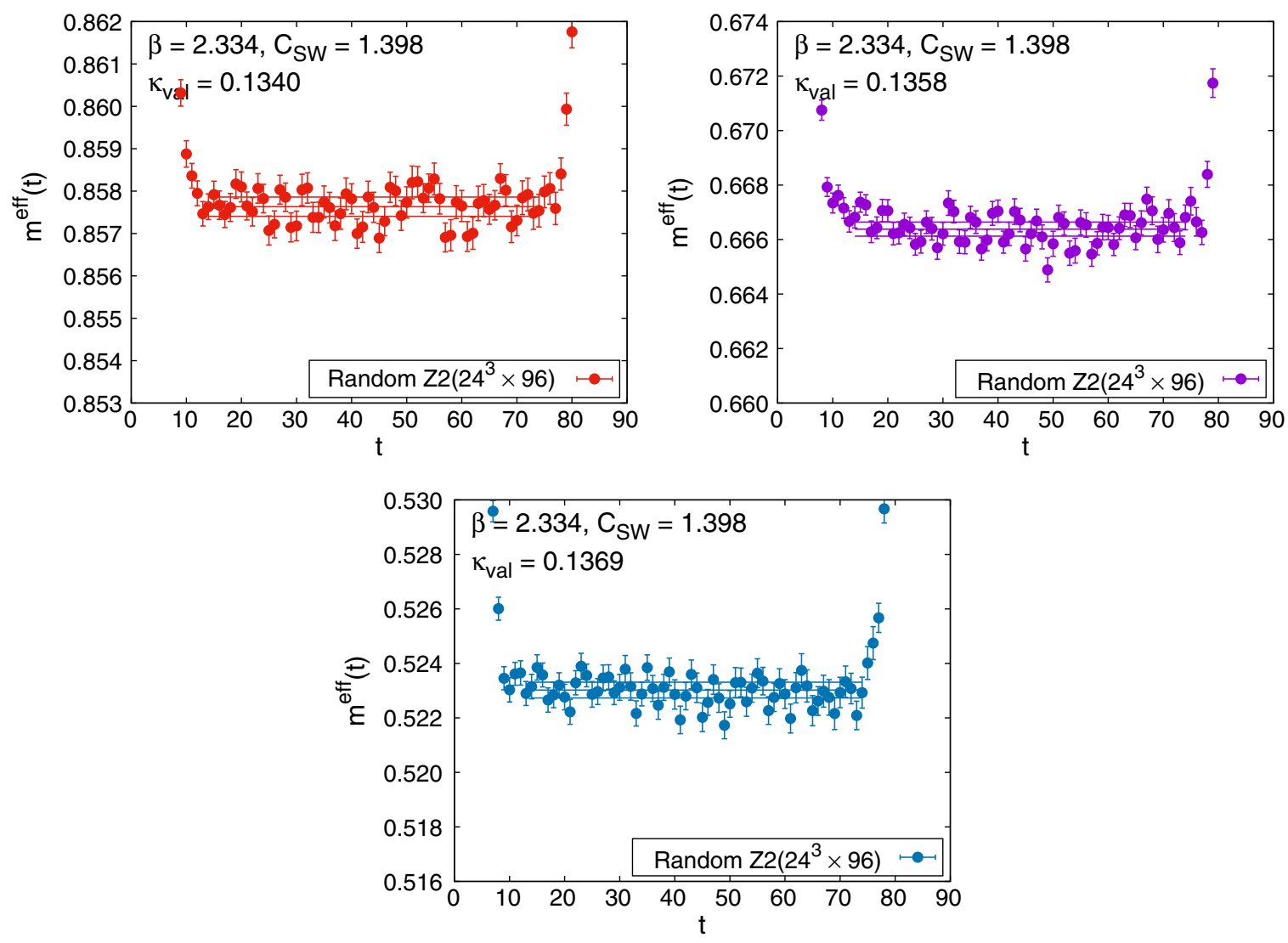

FIG. 1. Effective masses of a single pion. Results of a single exponential fit with $1 \sigma$ error are denoted by the solid lines. 
TABLE II. $\quad m_{\pi}$ and $E_{k}$ on $24^{3} \times 96$.

\begin{tabular}{lcc}
\hline \hline$\kappa_{\text {val }}$ & $m_{\pi}[\mathrm{GeV}]$ & $E_{k}[\mathrm{GeV}]$ \\
\hline 0.1340 & $0.85763(23)$ & $1.71703(47)$ \\
0.1358 & $0.66638(26)$ & $1.33514(52)$ \\
0.1369 & $0.52302(29)$ & $1.04889(60)$ \\
\hline \hline
\end{tabular}

setup, we found the gain is roughly a factor of 3 . We also calculate a single pion correlator $C_{\pi}(t)$ with the same source to measure $m_{\pi}$ in the large time region, $t \gg 1$,

$C_{\pi}(t)=\frac{1}{N_{r}} \sum_{i=1}^{N_{r}} \sum_{\mathbf{x}}\left\langle 0\left|\pi^{+}\left(\mathbf{x}, t_{\text {sink }}\right)\left(\pi^{+}\left(t_{\text {src }}, \eta_{i}\right)\right)^{\dagger}\right| 0\right\rangle$.

We perform the measurements with every fourth time slice per configuration; i.e., the total number of $t_{\mathrm{src}}$ is 24 . We adopted the periodic boundary condition in space and the Dirichlet boundary condition in time. Distance between the Dirichlet boundary and $t_{\mathrm{src}}$ is kept to be 12 .

We employ two methods to determine the interaction momentum $k$. One is a momentum from $E_{k}$, denoted by $k_{t}$,

$$
k_{t}^{2}=\frac{E_{k}^{2}}{4}-m_{\pi}^{2} .
$$

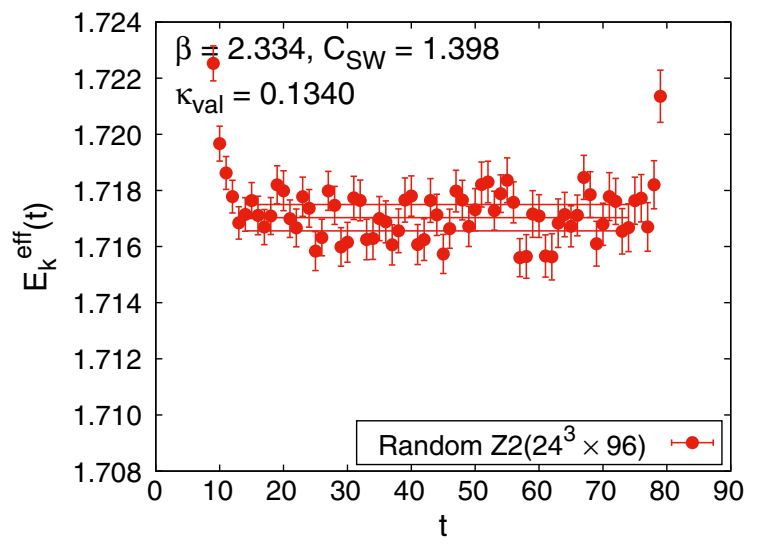

$E_{k}$ is obtained from the temporal correlator of two pions using $C_{\pi \pi}(\mathbf{x}, t)$ in Eq. (6),

$$
C_{\pi \pi}(t)=\sum_{\mathbf{x}} C_{\pi \pi}(\mathbf{x}, t)
$$

The other is a momentum from the BS wave function $\phi(\mathbf{x} ; k)$ outside the interaction range, denoted by $k_{s}$,

$$
k_{s}^{2}=-\frac{\Delta \phi(\mathbf{x} ; k)}{\phi(\mathbf{x} ; k)}, \quad x>R .
$$

The condition Eq. (10) is satisfied by definition.

\section{RESULT}

\section{A. Effective mass and energy}

Figure 1 represents our results of effective masses of a single pion, defined by

$$
m^{\mathrm{eff}}(t)=\log \left(\frac{C_{\pi}(t)}{C_{\pi}(t+1)}\right) .
$$

A plateau of the effective mass starts from $t=14$ in all $\kappa_{\mathrm{val}}$ cases. We determine $m_{\pi}$ from a single exponential fit to

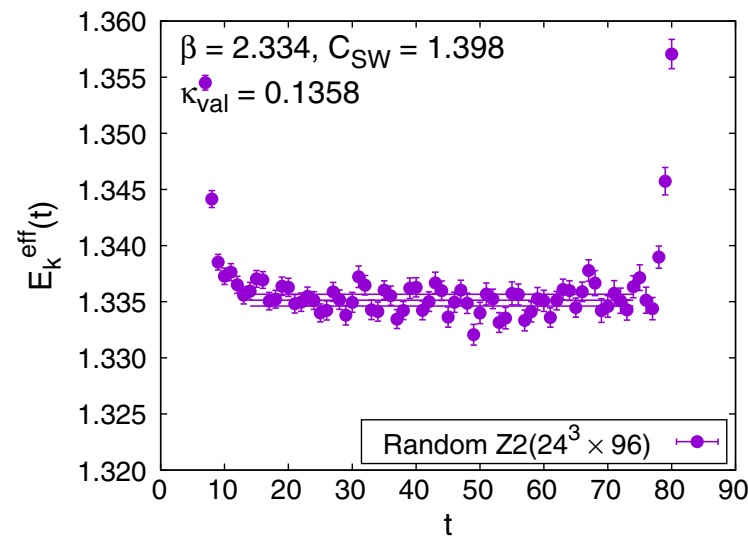

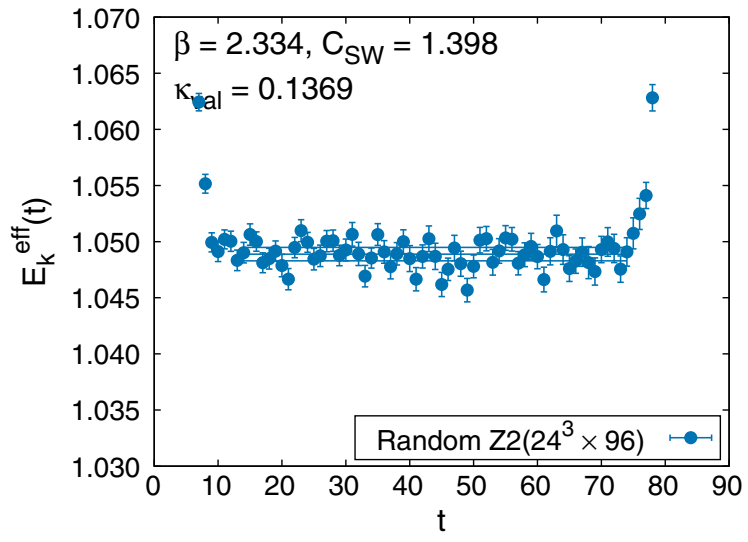

FIG. 2. Effective energies of $I=2$ two pions. Results of a single exponential fit with $1 \sigma$ error are denoted by the solid lines. 
TABLE III. $\quad k_{t}^{2}$ and $k_{s}^{2}$ on $24^{3} \times 96$.

\begin{tabular}{lcc}
\hline \hline$\kappa_{\text {val }}$ & $k_{t}^{2}\left[\mathrm{GeV}^{2}\right]$ & $k_{s}^{2}\left[\mathrm{GeV}^{2}\right]$ \\
\hline 0.1340 & $1.513(54) \times 10^{-3}$ & $1.549(20) \times 10^{-3}$ \\
0.1358 & $1.582(48) \times 10^{-3}$ & $1.519(19) \times 10^{-3}$ \\
0.1369 & $1.488(48) \times 10^{-3}$ & $1.497(23) \times 10^{-3}$ \\
\hline \hline
\end{tabular}

$C_{\pi}(t)$ in the range of $\left[t_{\min }, t_{\max }\right]=[14,74]$. The values of $m_{\pi}$ are listed in Table II.

Figure 2 plots effective energies of $I=2$ two pions defined by

$$
E_{k}^{\mathrm{eff}}(t)=\log \left(\frac{C_{\pi \pi}(t)}{C_{\pi \pi}(t+1)}\right)
$$

We employ the same temporal fitting procedure as that of a single pion to determine an interaction energy of two pions. The fitted values of $E_{k}$ is summarized in Table II.

Using the results for $m_{\pi}$ and $E_{k}$ obtained from the fits, $k_{t}^{2}$ in Eq. (17) is evaluated. The values of $k_{t}^{2}$ are tabulated in Table III.

\section{B. BS wave function}

The BS wave function $\phi(\mathbf{x} ; k)$ in Eq. (3) is calculated as follows. An effective BS wave function is defined by

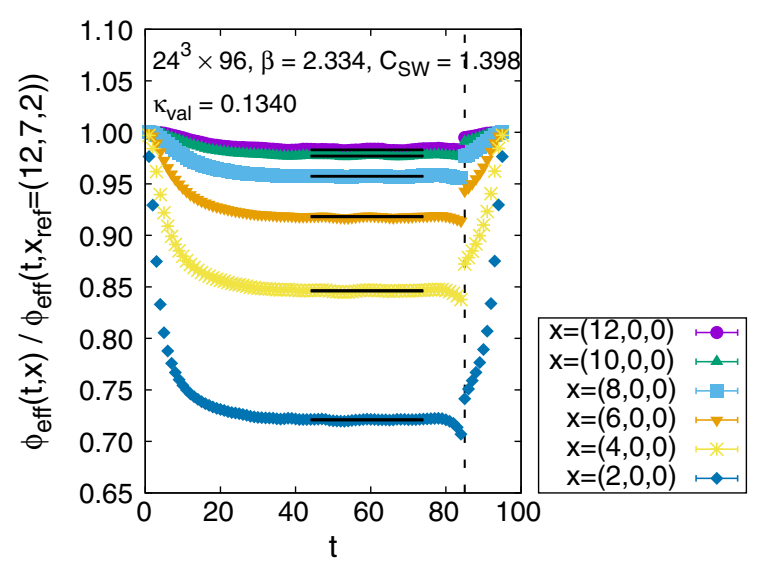

$$
\frac{\phi_{\text {eff }}(t, \mathbf{x})}{\phi_{\text {eff }}\left(t, \mathbf{x}_{\mathrm{ref}}\right)}=\frac{C_{\pi \pi}(t, \mathbf{x})}{C_{\pi \pi}\left(t, \mathbf{x}_{\mathrm{ref}}\right)}
$$

We choose the reference position of $\mathbf{x}_{\mathrm{ref}}=(12,7,2)$. $\phi_{\text {eff }}(t, \mathbf{x}) / \phi_{\text {eff }}\left(t, \mathbf{x}_{\text {ref }}\right)$ is plotted in Fig. 3. $\phi_{\text {eff }}(t, \mathbf{x}) /$ $\phi_{\text {eff }}\left(t, \mathbf{x}_{\text {ref }}\right)$ monotonically decreases with $t$ in an early $t$ region, where excited state contributions are clearly seen. A longer time separation is needed for the BS wave functions than those for a pion mass and two-pion energy. Boundary effects are also observed in the large $t$ region near the Dirichlet boundary position. The plateau of BS wave functions is observed in $t=44-74$. We extract $\phi(\mathbf{x} ; k)$ by a constant fit to $\phi_{\text {eff }}(t, \mathbf{x})$ in all $\mathbf{x}$ combined with the single exponential fit in the range of $\left[t_{\min }, t_{\max }\right]=[44,74]$.

\section{Sufficient condition}

We confirm the sufficient condition of Eq. (11) is satisfied in our simulation. The reduced BS wave function $h(\mathbf{x} ; k)$ in Eq. (8) is calculated using the fit result of $\phi_{\text {eff }}(t, \mathbf{x})$, and $k_{t}^{2}$ tabulated in Table III. Figure 4 illustrates our results of $h(\mathbf{x} ; k)$. We employ a ratio of $h(\mathbf{x} ; k)$ over $\phi\left(\mathbf{x}_{\text {ref }} ; k\right)$ at a reference point $\mathbf{x}_{\text {ref }}$ to cancel out the overall factor. For $x \gtrsim 10, h\left(\mathbf{x} ; k_{t}\right)=0$ is found to be satisfied in our statistical precision. This result shows the interaction range $R \sim 10<L / 2$, and the exponential tail of $h(\mathbf{x} ; k)$ is
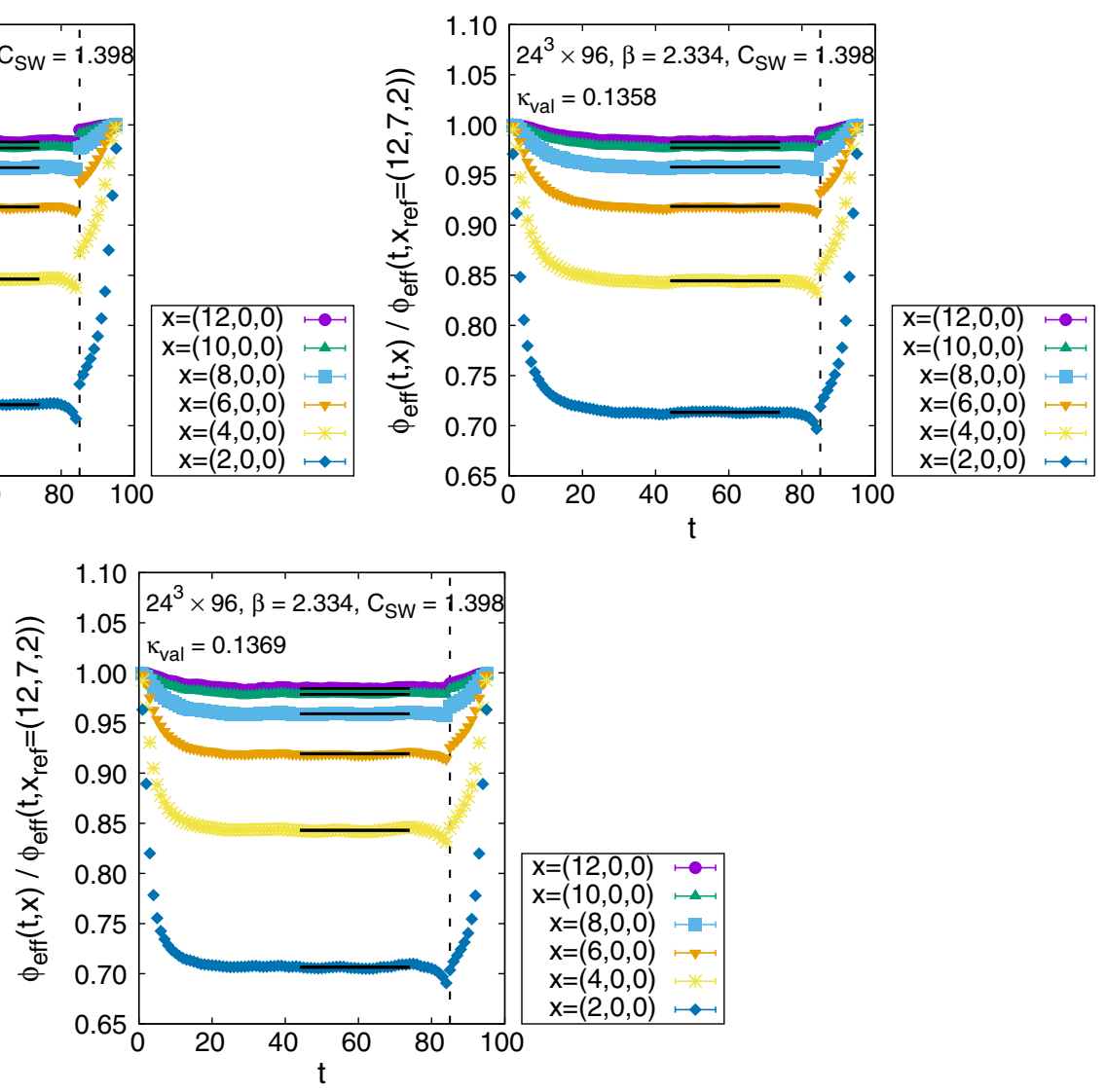

FIG. 3. Ratios of effective BS wave functions of two pions with the fit results represented by solid horizontal lines. The vertical dotted line denotes the Dirichlet boundary position. 

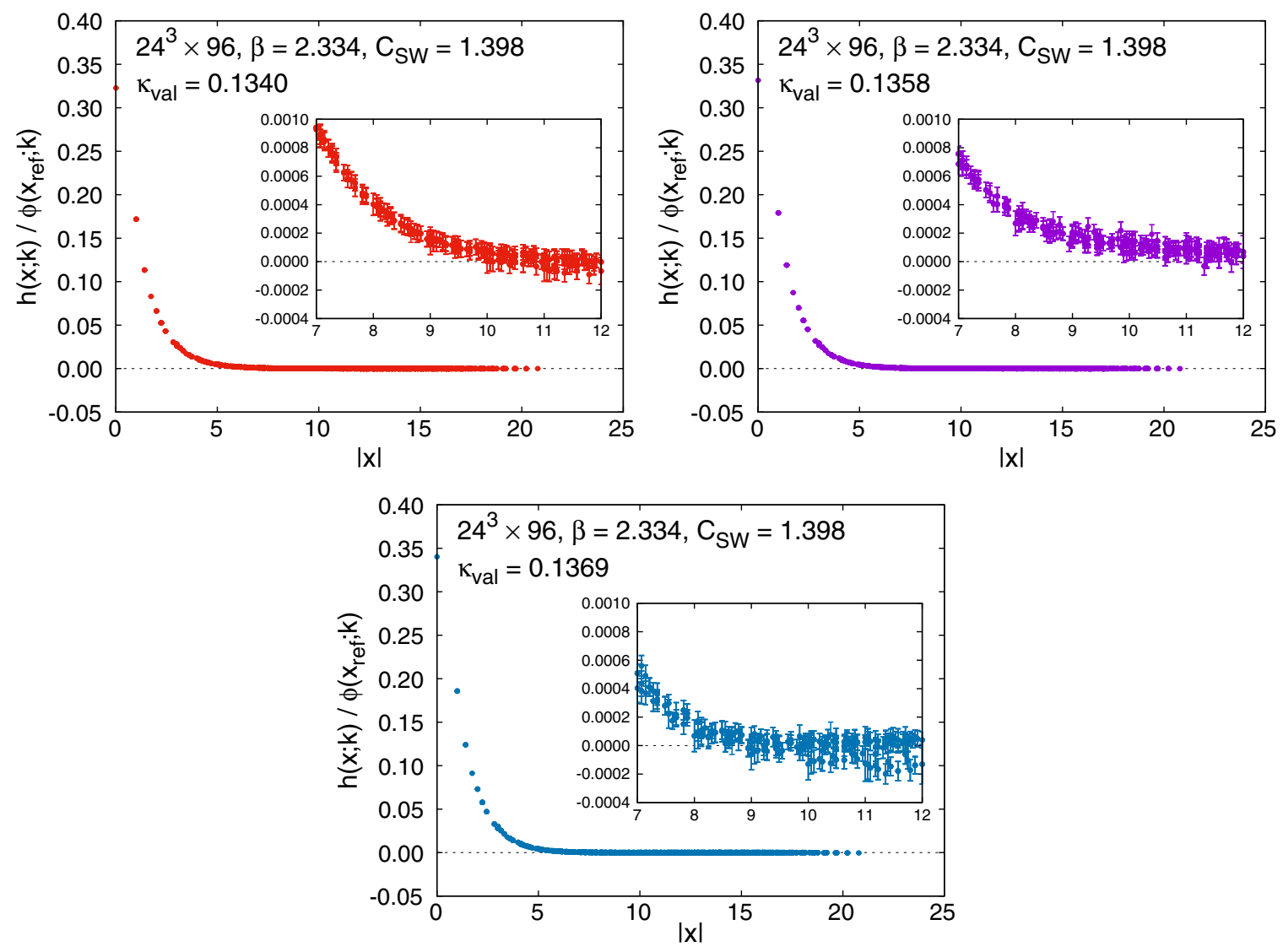

FIG. 4. Ratio of the reduced wave function $h(\mathbf{x} ; k)$ over the wave function $\phi\left(\mathbf{x}_{\text {ref }} ; k\right)$ at a reference point $\mathbf{x}_{\text {ref }}=(12,7,2)$ using $k=k_{t}$. The inside panel enlarges data in $7 \leq x \leq 12$.

negligible compared to our statistical error. Our data guarantee the sufficient condition of Eq. (11) in our quark mass region.

Using data in the outside region of $R$, an alternative interacting momentum $k_{s}^{2}$ in Eq. (19) can be determined, which is more precise than $k_{t}^{2}$ [6]. We obtain $k_{s}^{2}$ from a constant fit to $-\Delta \phi(\mathbf{x} ; k) / \phi(\mathbf{x} ; k)$ with the fit range of $\left[x_{\min }, x_{\max }\right]=[10,12 \sqrt{3}]$. Table III collects our results of $k_{s}^{2}$, as well as $k_{t}^{2} . k_{s}^{2}$ is consistent with $k_{t}^{2}$ with a smaller error than that of $k_{t}^{2}$ by a factor of 2 .

\section{Scattering amplitude}

Once the sufficient condition $R \sim 10<L / 2$ is satisfied, the scattering amplitude can be computed using Eq. (11). We choose $k=k_{s}$ in the following analyses, unless explicitly stated.

Figure 5 displays off-shell momentum dependence of the half off-shell amplitude $H(p ; k)$ in Eq. (12). The overall factors of $H_{L}(p ; k)$ in Eq. (11) are eliminated by taking a ratio of $H_{L}(p ; k)$ over its on-shell value $H_{L}(k ; k)$,

$$
\frac{H(p ; k)}{H(k ; k)}=\frac{H_{L}(p ; k)}{H_{L}(k ; k)} .
$$

A clean signal of the ratio is observed throughout our $p^{2}$ range. The validity of $H(p ; k)$ is ensured below the threshold drawn in the figure at $p^{2}=3 m_{\pi}^{2}$, i.e., $E_{k}=4 m_{\pi}$, though the quenched approximation prohibits dynamical inelasticity.

The operator dependence of $H_{L}(p ; k)$ is examined for both source and sink operators. The dependence is under

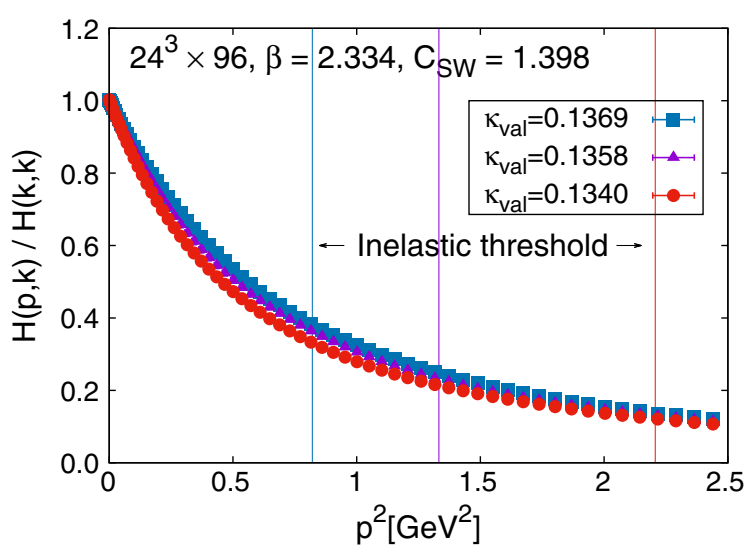

FIG. 5. Momentum dependence of a ratio of the scattering amplitude at half off-shell $H(p ; k)$ over the value at on-shell $H(k ; k)$. The vertical line expresses the threshold momentum of the two-pion scattering. 
control in our simulations. The details are explained in Appendix A.

We discuss the lattice artifacts in our result of $H_{L}(p ; k)$. The rotational symmetry breaking at the finite lattice spacing causes deviation between on axis and off axis $h(\mathbf{x} ; k)$ values. The influence to $H_{L}(p ; k)$ is evaluated to be $3 \%$ in our simulation at $a^{-1}=1.207 \mathrm{GeV}$. The size of the error is comparable to our statistical error. Another issue is the finite lattice artifact in the short distance. It highly affects the data, especially around $x=0$. They are suppressed in $H_{L}(p ; k)$, however, due to the Jacobian factor $r^{2}$ in the integration,

$$
\begin{aligned}
H_{L}(p ; k) & =-\sum_{i=1}^{3} \sum_{x_{i}=-L / 2+1}^{L / 2} C_{k} h(\mathbf{x} ; k) j_{0}(p r) \\
& \simeq-4 \pi \int_{0}^{\sqrt{3} L / 2} d r r^{2} C_{k} h(\mathbf{x} ; k) j_{0}(p r) .
\end{aligned}
$$

Contribution from $h(\mathbf{x} ; k)$ near $x=0$ is not significant. Nevertheless, the continuum limit is required to remove these lattice artifacts.

$H_{L}(p ; k)$ can be also calculated with the BS wave function in the momentum space, $\tilde{\phi}(p ; k)=\sum_{\mathbf{x}} \phi(\mathbf{x} ; k) e^{-i \mathbf{p} \cdot \mathbf{x}}$ (see, e.g., Ref. [10]). We numerically confirmed both approaches give a consistent value. Appendix B explains details of the formulation in the momentum space.

Two supplemental confirmations of the validity of $R \sim 10$ are possible by use of the scattering amplitude. One is the range of the summation for $H_{L}(p ; k)$ in Eq. (11). $H_{L}(p ; k)$ using a summation over all spatial volume gives a consistent value with that using a summation up to $x=10 \sim R$, which implies correctness of the estimate of $R \sim 10$. The other utilizes the on-shell scattering amplitude $H_{L}(k ; k)$ and an analytic solution of $\left(\Delta+k^{2}\right) \phi(\mathbf{x} ; k)=0$ in $x>R$. In the $S$-wave case, the analytic solution $\phi_{x>R}(\mathbf{x} ; k)$ can be expressed using the Green function on the lattice $G(\mathbf{x} ; k)$,

$$
\begin{aligned}
C_{k} \phi_{x>R}(\mathbf{x} ; k) & =v_{00} G(\mathbf{x} ; k), \\
G(\mathbf{x} ; k) & =\frac{1}{L^{3}} \sum_{\mathbf{p} \in \Gamma} e^{i \mathbf{x} \cdot \mathbf{p}} \frac{1}{p^{2}-k^{2}}, \\
\Gamma & =\left\{\mathbf{p} \mid \mathbf{p}=\frac{2 \pi}{L} \mathbf{n}, \mathbf{n} \in \mathbf{Z}^{3}\right\},
\end{aligned}
$$

where $v_{00}$ is a constant. $\phi_{x>R}(\mathbf{x} ; k)$ can be also expressed by the phase shift $\delta(k)$,

$$
C_{k} \phi_{x>R}(\mathbf{x} ; k)=C_{00} e^{i \delta(k)} \frac{\sin (k x+\delta(k))}{k x}+(l \geq 4),
$$

where $(l \geq 4)$ contains only the spherical Bessel functions $j_{l}(k x)$ of $l \geq 4$. Comparing Eq. (26) with Eq. (29) using the expansion by $j_{0}(k x)$ and $l=0$ spherical Neumann function $n_{0}(k x)$ leads to two simple equations [6]. The coefficient of $n_{0}(k x)$ gives

$$
H_{L}(k ; k)=v_{00}
$$

In parallel, the coefficient of $j_{0}(k x)$ provides

$$
k \cot \delta(k) H_{L}(k ; k)=4 \pi v_{00} g_{00}(k),
$$

where

$$
g_{00}(k)=\frac{1}{L^{3}} \sum_{\mathbf{p} \in \Gamma} \frac{1}{p^{2}-k^{2}} .
$$

Using Eqs. (30) and (31), one obtains the finite volume formula [4],

$$
k \cot \delta(k)=4 \pi g_{00}(k) .
$$

Therefore, Eq. (30) must be satisfied in the finite volume method. Based on this argument, we define an indicator $R(\mathbf{x} ; k)$ to test the equality in Eq. (30),

$$
R(\mathbf{x} ; k)=\frac{H_{L}(k ; k)}{C_{k} \phi(\mathbf{x} ; k)} G(\mathbf{x} ; k) .
$$

Outside the interaction range $R, R(\mathbf{x} ; k)$ becomes unity, if Eq. (30) is satisfied. Figure 6 represents our result of $R(\mathbf{x} ; k)$. It increases monotonically and approaches to unity. $R(\mathbf{x} ; k)$ is consistent with unity in $x \gtrsim 10$, as expected. It validates our estimate of $R \sim 10$.

We also discuss $t$ dependence of the on-shell amplitude $H_{L}(k ; k) . H_{L}(k ; k)$ is calculated at each $t$ for the $t$ dependence analysis, in contrast to the above calculation using the fit result in Sec. IV B. Figure 7 illustrates $t$ dependence of $H_{L}(k ; k) /\left(C_{k} \phi\left(\mathbf{x}_{\text {ref }} ; k\right)\right)$. The ratio is almost flat in $t$. Figure 8 displays, on the other hand, the numerator and denominator at $\kappa_{\mathrm{val}}=0.1340$ multiplied by the exponential factor of the two-pion ground state energy $e^{E_{k} t}$. The results

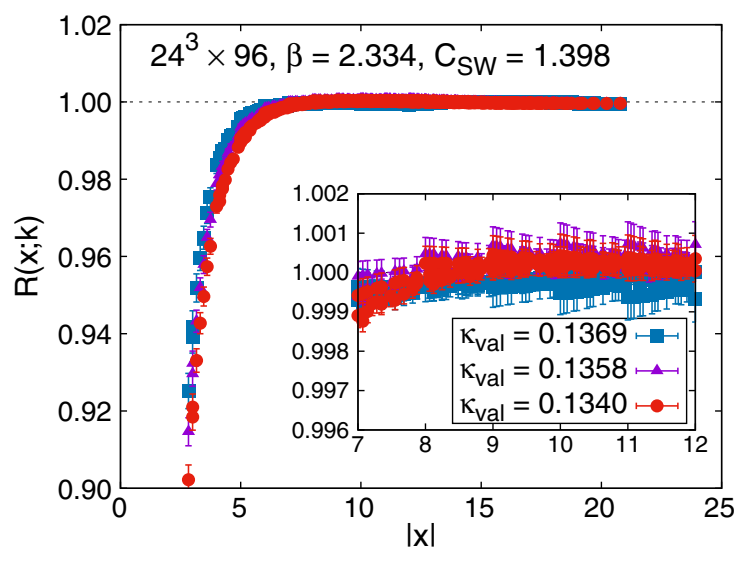

FIG. 6. $\quad \mathbf{x}$ dependence of the ratio $R(\mathbf{x} ; k)$ defined in Eq. (34). 


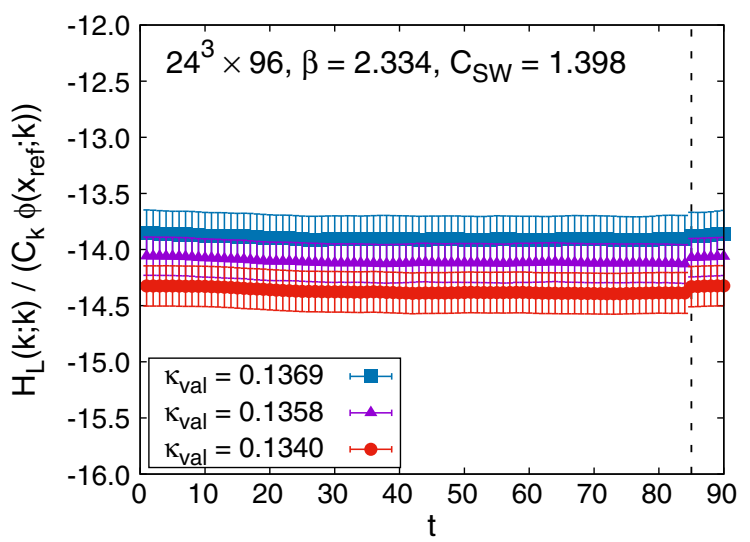

FIG. 7. $t$ dependence of the scattering amplitude over the wave function $H_{L}(k ; k) /\left(C_{k} \phi\left(\mathbf{x}_{\mathrm{ref}} ; k\right)\right)$ with $\mathbf{x}_{\text {ref }}=(12,7,2)$. The vertical dotted line denotes the Dirichlet boundary position.

show clear excited state contributions in the small $t$ region, $t<10$. It should be noticed that a choice of $x_{\text {ref }}$ varies the excited state contributions of the denominator, as indicated in Fig. 3. Figures 7 and 8 suggest contributions from the excited states are well compensated in the ratio of $H_{L}(k ; k) /\left(C_{k} \phi\left(\mathbf{x}_{\text {ref }} ; k\right)\right)$. The details are discussed in Appendix C. Further investigation of the excited state compensation needs the variational method.

\section{E. Physical quantities from scattering amplitudes}

We can extract physical observables from the scattering amplitude. The scattering phase shift $\delta(k)$ is obtained by the scattering amplitude at on-shell $H_{L}(k ; k)$ and the BS wave function at some reference point outside of the interaction range $x_{\text {ref }}>R$,

$$
\frac{H_{L}(k ; k)}{C_{k} \phi\left(\mathbf{x}_{\mathrm{ref}} ; k\right)}=\frac{4 \pi x_{\mathrm{ref}} \sin \delta(k)}{\sin \left(k x_{\mathrm{ref}}+\delta(k)\right)} .
$$

We used the expansion of $C_{k} \phi\left(\mathbf{x}_{\text {ref }} ; k\right)$ in Eq. (29) and assumed $l \geq 4$ terms are negligible. The phase factor as

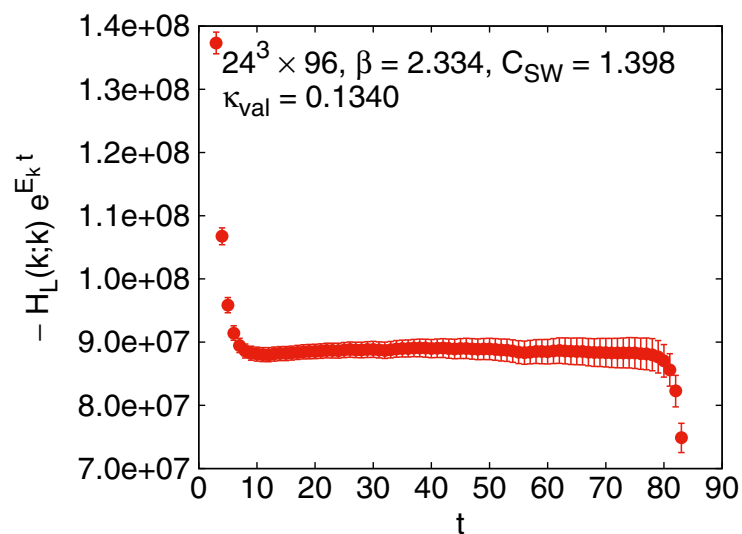

well as the overall constants are canceled in the ratio. Inversely, $\delta(k)$ is given by

$$
\tan \delta(k)=\frac{\sin \left(k x_{\mathrm{ref}}\right)}{4 \pi x_{\mathrm{ref}} \frac{C_{k} \phi\left(\mathbf{x}_{\mathrm{ref}} ; k\right)}{H_{L}(k ; k)}-\cos \left(k x_{\mathrm{ref}}\right)} .
$$

The reference point is chosen to be $\mathbf{x}_{\text {ref }}=(12,7,2)$ by the following procedure. Evaluation of $\tan \delta(k)$ through Eq. (36) requires the $l \geq 4$ terms in Eq. (29) must be negligible at $\mathbf{x}_{\text {ref }}$. We select $\mathbf{x}_{\text {ref }}$ to minimize the leading $l=4$ contribution in the $(l \geq 4)$ terms. The size of the $l=4$ term is examined using an expansion of $\phi(\mathbf{x} ; k)$ in $x>R$,

$$
\begin{aligned}
\phi(\mathbf{x} ; k)= & A_{0}(k) Y_{00}\left(R_{A_{1}^{+}}[\mathbf{x} / x]\right) n_{0}(k x) \\
& +B_{0}(k) Y_{00}\left(R_{A_{1}^{+}}[\mathbf{x} / x]\right) j_{0}(k x) \\
& +B_{4}(k) Y_{40}\left(R_{A_{1}^{+}}[\mathbf{x} / x]\right) j_{4}(k x) \\
& +(l \geq 6),
\end{aligned}
$$

where $A_{l}(k), B_{l}(k)$ are constants. $Y_{l m}(\mathbf{x} / x)$ is the spherical harmonic function with $A_{1}^{+}$projector, $R_{A_{1}^{+}}$. It is an alternative expression of Eq. (29). Assuming $A_{l}(k), B_{l}(k)=$ $O(1)$, the size of the $l=4$ contribution at each $\mathbf{x}$ is estimated by using a ratio $Y(\mathbf{x} ; k)$,

$$
Y(\mathbf{x} ; k)=\frac{Y_{40}\left(R_{A_{1}^{+}}[\mathbf{x} / x]\right) j_{4}(k x)}{Y_{00}\left(R_{A_{1}^{+}}[\mathbf{x} / x]\right) j_{0}(k x)} .
$$

$Y(\mathbf{x} ; k)$ with $k=k_{t}$ at $\kappa_{\text {val }}=0.1340$ is presented in Fig. 9. The values of $Y(\mathbf{x} ; k)$ at some positions in $x>10$ are found to be close to zero, satisfying $|Y(\mathbf{x} ; k)|<10^{-6}$. Similar results of $Y(\mathbf{x} ; k)$ are obtained in other $\kappa_{\mathrm{val}}$. From the estimation, we choose a reference point as $\mathbf{x}_{\text {ref }}=(12,7,2)$.

The effective range expansion defines the scattering length $a_{0}$ and the effective range $r_{\text {eff }}$,

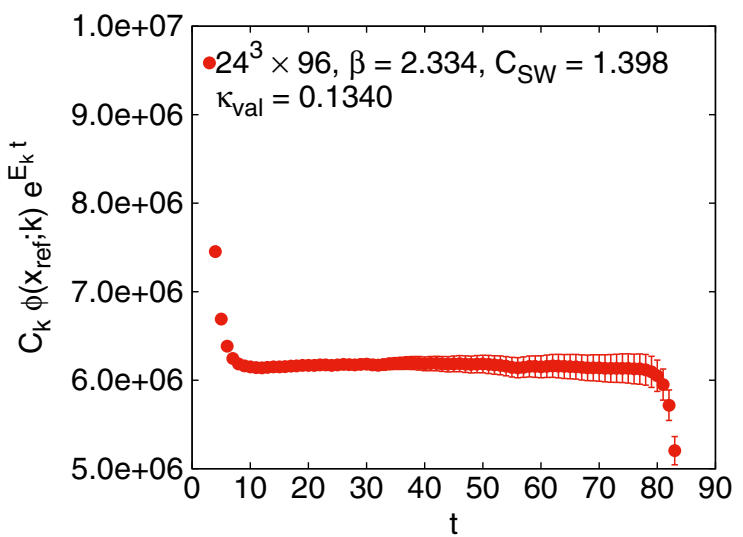

FIG. 8. Numerator and denominator of $H_{L}(k ; k) /\left(C_{k} \phi\left(\mathbf{x}_{\text {ref }} ; k\right)\right)$ at $\kappa_{\text {val }}=0.1340$ multiplied by $e^{E_{k} t}$ are plotted in the left and right panels. 


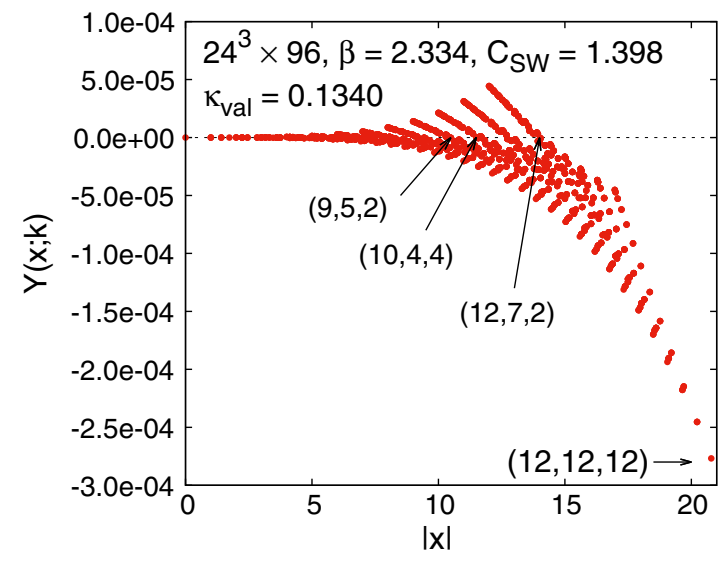

FIG. 9. $\quad Y(\mathbf{x} ; k)$ in Eq. (38) at $\kappa_{\mathrm{val}}=0.1340$ as a function of $|\mathbf{x}|$. An arrow expresses three components of each $\mathbf{x}$.

$$
\frac{k}{\tan \delta(k)}=\frac{1}{a_{0}}+r_{\mathrm{eff}} k^{2}+O\left(k^{4}\right) .
$$

We estimate $a_{0}$ by

$$
a_{0}=\frac{\tan \delta(k)}{k}
$$

The tiny value of $k^{2}$ presented in Table III justifies the estimation. Similarly, $r_{\text {eff }}$ can be evaluated by

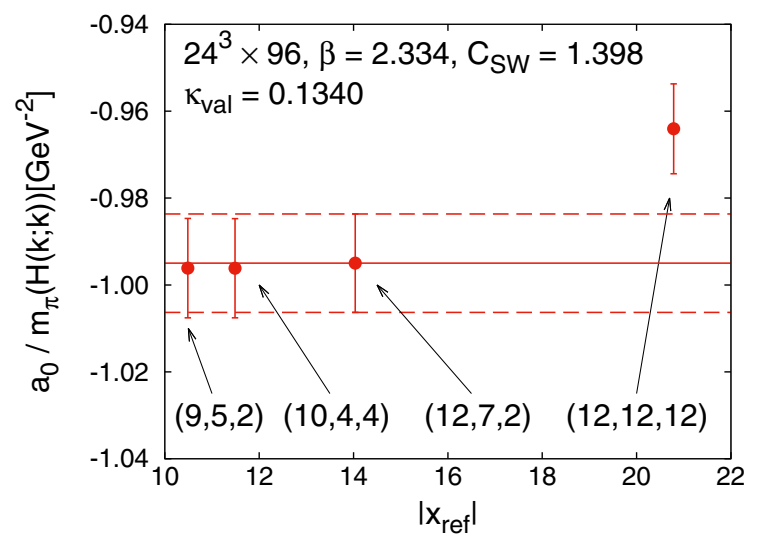

$$
\begin{gathered}
r_{\mathrm{eff}}=-\frac{2 k^{2} H^{\prime} / H_{L}(k ; k)+\sin ^{2} \delta(k)}{2 k \sin \delta(k) \cos \delta(k)}, \\
H^{\prime}=\left.\frac{\partial H_{L}(p ; k)}{\partial p^{2}}\right|_{p^{2}=k^{2}},
\end{gathered}
$$

where we assume

(1) The phase of $H(p ; k)$ is $e^{i \delta(k)}$ at $p^{2} \approx k^{2}$

(2) $\left.\frac{\partial H(p ; k) e^{-i \delta(k)}}{\partial p^{2}}\right|_{p^{2}=k^{2}}=\left.\frac{\partial H(p ; p) e^{-i \delta(p)}}{\partial p^{2}}\right|_{p^{2}=k^{2}}$.

\section{Scattering length}

We evaluate $a_{0} / m_{\pi}$ through Eq. (40) using $\tan \delta(k)$ obtained from Eq. (36). In the evaluation, not only $k=k_{s}$ but also $k=k_{t}$ is employed. A smaller error of $a_{0} / m_{\pi}$ is obtained by $k=k_{s}$. The results are tabulated in Table IV.

Since $\tan \delta(k)$ in Eq. (36) depends on the choice of the reference point $x_{\text {ref }}, x_{\text {ref }}$ dependence of $a_{0} / m_{\pi}$ is also investigated. Figure 10 exhibits $x_{\text {ref }}$ dependence of $a_{0} / m_{\pi}$ at each $\kappa_{\mathrm{val}}$. The left two data are obtained with the reference positions, $\mathbf{x}_{\text {ref }}=(9,5,2)$ and $(10,4,4)$, which satisfy the same condition $|Y(\mathbf{x} ; k)|<10^{-6}$ as $\mathbf{x}_{\text {ref }}=$ $(12,7,2)$, expressed in Fig. 9. These data are consistent with each other. Contrarily, the farthest right point in Fig. $10, \mathbf{x}_{\text {ref }}=(12,12,12)$, overestimates the values from

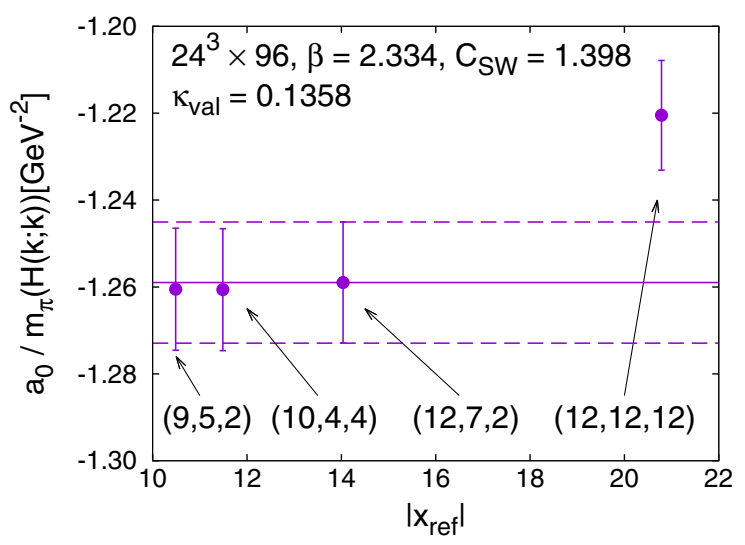

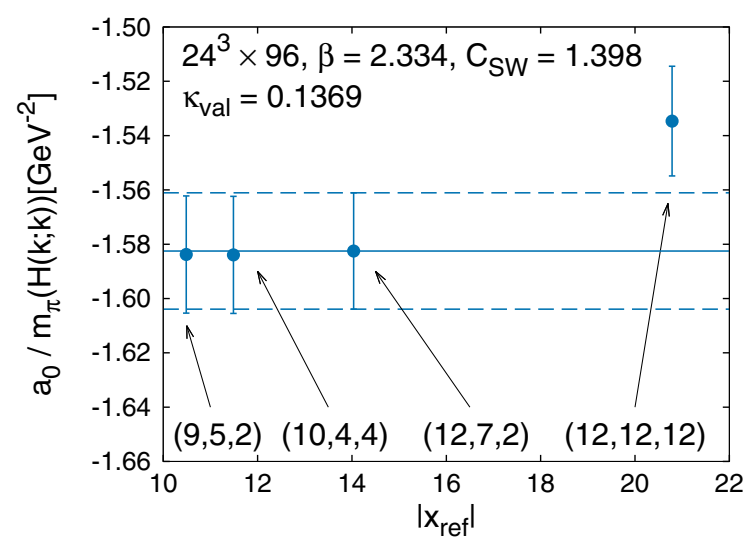

FIG. 10. Reference point $\mathbf{x}_{\text {ref }}$ dependence of $a_{0} / m_{\pi}$ obtained from $H(k ; k)$. The solid line represents the result with $\mathbf{x}_{\text {ref }}=(12,7,2)$ with $1 \sigma$ error band. 
TABLE IV. Scattering length $a_{0}$ over the pion mass $m_{\pi}$ obtained with $k_{t}$ and $k_{s}$ on $24^{3} \times 96$.

\begin{tabular}{lcc}
\hline \hline$\kappa_{\text {val }}$ & $a_{0} / m_{\pi}\left(k_{t}\right)\left[\mathrm{GeV}^{-2}\right]$ & $a_{0} / m_{\pi}\left(k_{s}\right)\left[\mathrm{GeV}^{-2}\right]$ \\
\hline 0.1340 & $-0.975(31)$ & $-0.995(11)$ \\
0.1358 & $-1.305(35)$ & $-1.259(14)$ \\
0.1369 & $-1.575(45)$ & $-1.582(21)$ \\
Physical & $-2.09(13)$ & $-2.30(8)$ \\
\hline \hline
\end{tabular}

the other reference positions beyond $1 \sigma$ error band. $\mathbf{x}_{\mathrm{ref}}=$ $(12,12,12)$ gives the largest value of $|Y(\mathbf{x} ; k)|$ as presented in Fig. 9. The result clearly indicates a sizable $l=4$ contribution at $\mathbf{x}_{\text {ref }}=(12,12,12)$. Our analysis of $x_{\text {ref }}$ dependence of $a_{0} / m_{\pi}$ suggests the $l=4$ contribution in $a_{0} / m_{\pi}$ is suppressed well by our choice of $\mathbf{x}_{\mathrm{ref}}=(12,7,2)$.

$a_{0} / m_{\pi}$ is extrapolated to the physical $m_{\pi}$ using a formula motivated by chiral perturbation theory [17],

$$
\frac{a_{0}}{m_{\pi}}=A_{a_{0}}+B_{a_{0}} m_{\pi}^{2}+C_{a_{0}} m_{\pi}^{4},
$$

where $A_{a_{0}}, B_{a_{0}}, C_{a_{0}}$ are fitting parameters. Our results at the physical point are listed in Table IV. Figure 11 summarizes chiral extrapolations of $a_{0} / m_{\pi}$. Our value at the physical point is consistent with the previous result by lattice QCD using the conventional finite volume method based on $\phi(\mathbf{x} ; k)$ outside the interaction range [6] and the phenomenological estimate [18]. The agreement ensures our approach with $\phi(\mathbf{x} ; k)$ inside the interaction range.

\section{Effective range}

The effective range $r_{\text {eff }}$ is evaluated by the slope of $H_{L}(p ; k)$ with respect to $p^{2}$ and $\delta(k)$ as shown in Eq. (41). Our results with $k=k_{s}$ and $k=k_{t}$ are compiled in Table V.

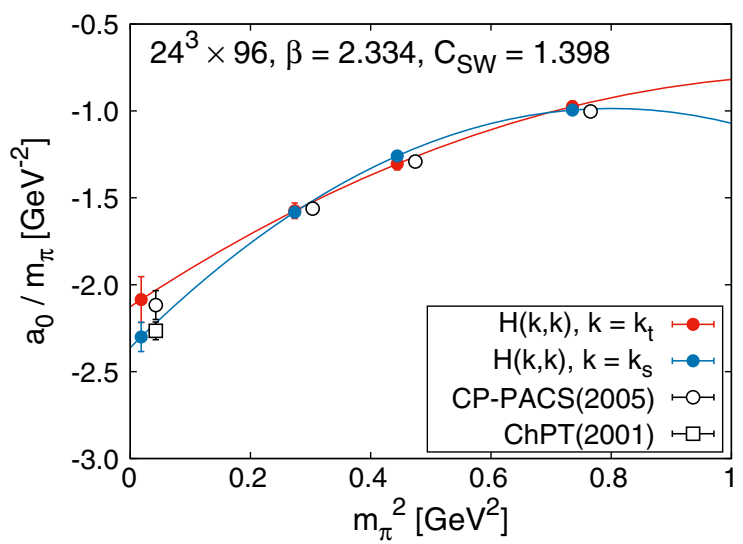

FIG. 11. Quark mass dependence of the ratio of the scattering length over the pion mass $a_{0} / m_{\pi}$. Open circles are lattice QCD results with the conventional finite volume method [6]. An open square is a phenomenological estimate of the chiral perturbation theory (ChPT) [18]. Open symbols are shifted to some extent for clarification of data.
TABLE V. $\quad$ Effective ranges $r_{\text {eff }}$ using $k_{t}$ and $k_{s}$ on $24^{3} \times 96$.

\begin{tabular}{lcc}
\hline \hline$\kappa_{\text {val }}$ & $r_{\text {eff }}\left(k_{t}\right)\left[\mathrm{GeV}^{-1}\right]$ & $r_{\text {eff }}\left(k_{s}\right)\left[\mathrm{GeV}^{-1}\right]$ \\
\hline 0.1340 & $-1.26(63)$ & $-1.63(22)$ \\
0.1358 & $-1.90(49)$ & $-1.28(25)$ \\
0.1369 & $-1.26(64)$ & $-1.36(28)$ \\
Physical & $10.8(7.6)$ & $10.6(4.1)$ \\
\hline
\end{tabular}

The result using $k_{t}$ has a larger error than that using $k_{s}$, as in the case of $a_{0}$. Figure 12 plots the $m_{\pi}$ dependence of $m_{\pi} r_{\text {eff }}$. Our result of $m_{\pi} r_{\text {eff }}$ agrees with the value obtained by the finite volume method, calculated using data in Ref. [6]. Our data are more accurate than those of the finite volume method due to the explicit $p^{2}$ dependence of $H(p ; k)$.

$r_{\text {eff }}$ is extrapolated to the physical point using a formula based on the chiral perturbation theory [20].

$$
m_{\pi} r_{\mathrm{eff}}=\frac{A_{r_{\mathrm{eff}}}}{m_{\pi}^{2}}+B_{r_{\mathrm{eff}}},
$$

where $A_{r_{\text {eff }}}, B_{r_{\text {eff }}}$ are fitting parameters. Our result at the physical point, whose value is summarized in Table V, underestimates the phenomenological value [18]. The reason seems to be the chiral extrapolation of $m_{\pi} r_{\text {eff }}$. It rapidly grows toward the physical point. We also need to validate the two assumptions in Eq. (41), though consistency between our result and that of the conventional finite volume method is confirmed at each simulation point. Another possibility is the quenching effect. In fact, $N_{f}=2+1$ lattice QCD using $m_{\pi}=390 \mathrm{MeV}$ successfully reproduces the phenomenological estimate [19]. More realistically $N_{f}=2+1$ data around the physical point are required to draw a definite conclusion.

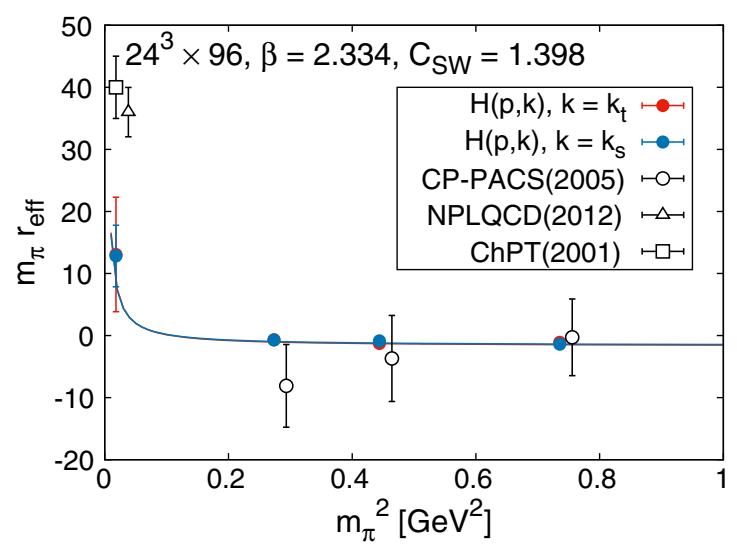

FIG. 12. Quark mass dependence of the effective range $r_{\text {eff }}$. Open circles are $N_{f}=0$ lattice QCD results with the conventional finite volume method calculated using data in Ref. [6]. An open triangle is the $N_{f}=2+1$ lattice QCD result with the finite volume method [19]. An open square is a phenomenological estimate of the chiral perturbation theory (ChPT) [18]. Open symbols are shifted to some extent for clarification of data. 


\section{SUMMARY}

We have successfully calculated the on-shell and half off-shell scattering amplitudes from the BS wave function inside the interaction range, as reported in our previous paper [9]. Our approach utilizes the BS wave function inside the interaction range, while the conventional finite volume method is based on the BS wave function outside the interaction range. The on-shell scattering amplitude gives direct scattering information through the phase shift. The half off-shell amplitude is not observable in experiments, but on the other hand, it could be an important input of theoretical effective theories and models to constrain their parameters. Furthermore, the half off-shell amplitude gives the effective range under two assumptions.

In this article, we extended our study to investigate the quark mass dependence of $I=2 \mathrm{~S}$-wave two-pion on-shell and half off-shell scattering amplitudes at the center of mass in the quenched QCD. Our simulation was performed at the lattice spacing of $a^{-1}=1.207 \mathrm{GeV}$ using pion masses of $m_{\pi}=0.52-0.86 \mathrm{GeV}$ on a $24^{3} \times 96$ lattice. We first checked the interaction range is within half of our spatial lattice, which satisfies a sufficient condition of our method as well as the finite volume method. It allows us to evaluate onshell and half off-shell scattering amplitudes. We obtained clean signals of them. We also discussed the source and sink operator independence of our scattering amplitudes.

We then extracted the scattering length from the on-shell scattering amplitude. Our results at each $m_{\pi}$ and the physical point agree with those obtained by the finite volume method. It proves our approach is an alternative to the conventional finite volume method.

We also extracted the effective range from the slope of the half off-shell amplitude at the on-shell momentum under two assumptions to be validated. Our result agrees with that by the conventional finite volume method at each pion mass. On the physical point, however, our result extrapolated from data with pion masses of $0.52-0.86 \mathrm{GeV}$ underestimates the recent lattice QCD and the phenomenological values. More realistic data near the physical point in $N_{f}=2+1$ lattice QCD are required to identify the reason of the underestimation.

The benefit of this approach is not only an on-shell but also a half off-shell scattering amplitude can be evaluated. Another benefit is that estimation of the scaling violation is possible by rotational symmetry breaking in $H_{L}(p ; k)$, which can not be evaluated in the conventional finite volume method.

An important future direction is application of our approach to more complicated systems, such as rho resonance and nonidentical hadron scatterings, $\pi-K, \pi-N$, etc.

\section{ACKNOWLEDGMENTS}

We thank J. Carbonell and V. A. Karmnov for pointing out the momentum space formulation, and members of
PACS Collaboration for useful discussion. We also appreciate INT at University of Washington for the kind hospitality and partial financial support during an early stage of this work. Our simulation was performed on COMA under Interdisciplinary Computational Science Program of Center for Computational Sciences, University of Tsukuba. This work is based on BRIDGE++ code (http://bridge.kek.jp/ Lattice-code/) [21]. This work is supported in part by JSPS KAKENHI Grants No. 16H06002 and No. 18K03638.

\section{APPENDIX A: OPERATOR DEPENDENCE OF SCATTERING AMPLITUDE}

We discuss the source and sink operator dependence of the scattering amplitude.

\section{Source operator dependence}

The source operator dependence is simply explained by an overall factor of $\phi(\mathbf{x} ; k)$. When the ground state dominates $C_{\pi \pi}(t)$ in a large $t$ region, the source operator dependence cancels in ratios, $\phi(\mathbf{x} ; k) / \phi\left(\mathbf{x}_{\text {ref }} ; k\right)$ and $h(\mathbf{x} ; k) / \phi\left(\mathbf{x}_{\text {ref }} ; k\right)$.

In order to check the source operator independence of $h(\mathbf{x} ; k) / \phi\left(\mathbf{x}_{\text {ref }} ; k\right)$, we compare results using the $\mathrm{Z} 2$ random and wall sources on the $24^{3} \times 64$ ensemble with $\kappa_{\mathrm{val}}=0.1340$, which was used partly in our previous paper [9]. The same simulation setup is adopted as that in Sec. III. We use 400(200) configurations with 32(16) measurements per configuration using the random $\mathrm{Z} 2$ (wall) source. The simulation parameters are listed in Table VI.

The wall source operator $\Omega_{\text {wall }}(t)$ in $C_{\pi \pi}(\mathbf{x}, t)$ of Eq. (6) is defined by

$$
\Omega_{\mathrm{wall}}(t)=\pi_{\mathrm{wall}}^{+}\left(t_{\mathrm{src}}\right) \pi_{\mathrm{wall}}^{+}\left(t_{\mathrm{src}}+1\right),
$$

where

$$
\pi_{\mathrm{wall}}^{+}\left(t_{\mathrm{src}}\right)=\left[\sum_{\mathbf{x}_{1}} \bar{d}\left(\mathbf{x}_{1}, t\right)\right] \gamma_{5}\left[\sum_{\mathbf{x}_{2}} u\left(\mathbf{x}_{2}, t\right)\right]
$$

The pion operators in $\Omega_{\text {wall }}(t)$ are placed at $t_{\text {src }}$ and $t_{\text {src }}+1$ to prevent from Fierz rearrangement [22,23].

Figure 13 exhibits the effective mass $m_{\pi}^{\text {eff }}$ and energy $E_{k}^{\text {eff }}$, defined in Eqs. (20) and (21), respectively. Our results from the two sources are consistent with each other, as well as that on a $24^{3} \times 96$ lattice. We fit data in the range of $\left[t_{\min }, t_{\max }\right]=[14,44]$ to extract $m_{\pi}$ and $E_{k}$. The results are summarized in Table VII together with those for $k_{t}^{2}$ and $k_{s}^{2}$.

TABLE VI. Simulation parameters on a $24^{3} \times 64$ lattice.

\begin{tabular}{lcccc}
\hline \hline Lattice size & $\kappa_{\text {val }}$ & $N_{\text {src }}$ & Source type & $N_{\text {config }}$ \\
\hline $24^{3} \times 64$ & 0.1340 & 32 & Z2 & 400 \\
& & 16 & Wall & 200 \\
\hline \hline
\end{tabular}



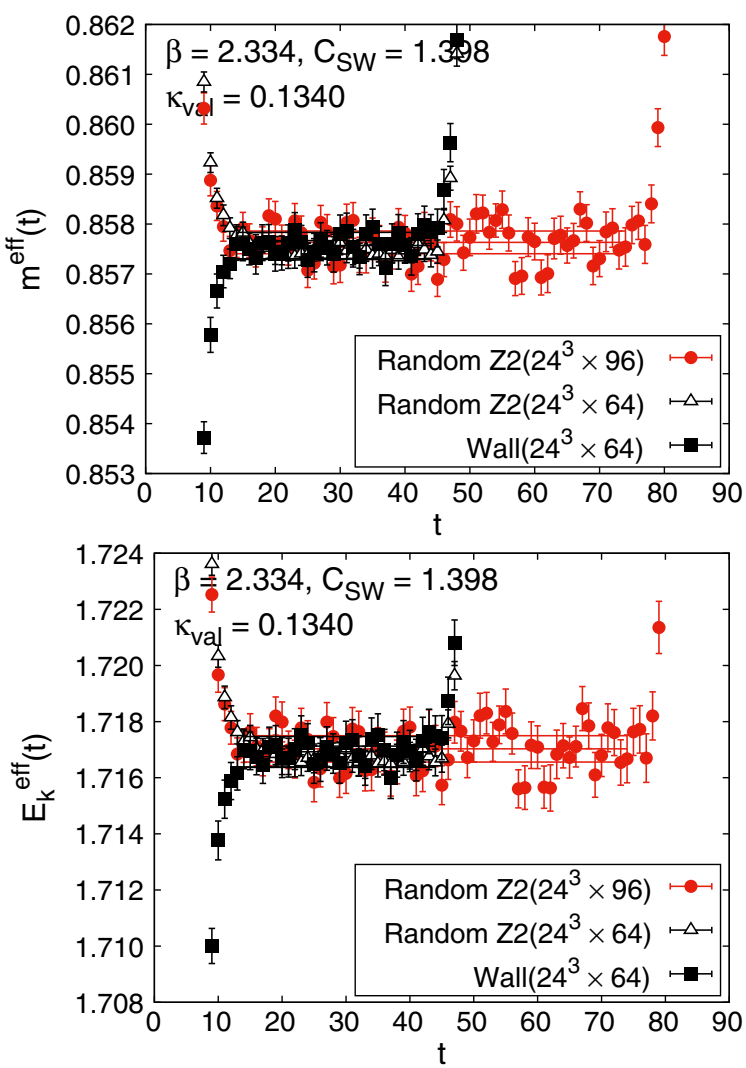

FIG. 13. Effective masses and two-pion energies with random $\mathrm{Z} 2$ and wall sources on a $24^{3} \times 64$ lattice. The data with random Z2 source on $24^{3} \times 96$ are also plotted.

In contrast to the case on a $24^{3} \times 96$ lattice in Fig. 3, the data of $\phi(\mathbf{x} ; k)$ on a $24^{3} \times 64$ lattice available for analysis of the BS wave function are limited to those at a time slice of $t=44$. We use $\phi(\mathbf{x} ; k)$ at $t=44$ for our analysis on a $24^{3} \times 64$ lattice.

Figure 14 presents the two source results of $h(\mathbf{x} ; k) /$ $\phi\left(\mathbf{x}_{\text {ref }} ; k\right)$. They agree with each other and with the result obtained on $24^{3} \times 96$ lattice in Fig. 4. Consistency of the two results proves the source independence of $h\left(\mathbf{x} ; k_{s}\right) / \phi\left(\mathbf{x}_{\text {ref }} ; k\right)$. We also confirmed the source operator independence of the physical quantities $a_{0} / m_{\pi}$ and $r_{\text {eff }}$ extracted from $H_{L}(p ; k)$. The results are in Table VII.

TABLE VII. $m_{\pi}, E_{k}, k_{t}^{2}, k_{s}^{2}$ and the physical quantities from scattering amplitudes on a $24^{3} \times 64$ lattice using random $\mathrm{Z} 2$ and wall sources at $k_{\mathrm{val}}=0.1340$.

\begin{tabular}{lcc}
\hline \hline Source & $\mathrm{Z} 2$ & Wall \\
\hline$m_{\pi}[\mathrm{GeV}]$ & $0.85748(19)$ & $0.85757(26)$ \\
$E_{k}[\mathrm{GeV}]$ & $1.71675(38)$ & $1.71693(54)$ \\
$k_{t}^{2}\left[\mathrm{GeV}^{2}\right]$ & $1.533(34) \times 10^{-3}$ & $1.535(56) \times 10^{-3}$ \\
$k_{s}^{2}\left[\mathrm{GeV}^{2}\right]$ & $1.569(27) \times 10^{-3}$ & $1.523(27) \times 10^{-3}$ \\
$a_{0} / m_{\pi}\left(k_{s}\right)\left[\mathrm{GeV}^{-2}\right]$ & $-0.963(10)$ & $-0.979(10)$ \\
$r_{\text {eff }}\left(k_{s}\right)\left[\mathrm{GeV}^{-2}\right]$ & $-1.85(10)$ & $-1.73(09)$ \\
\hline \hline
\end{tabular}

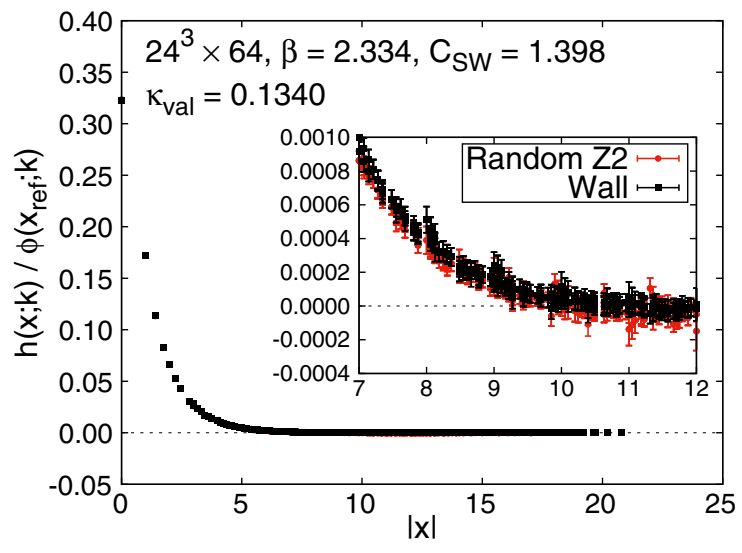

FIG. 14. Comparison of $h(\mathbf{x} ; k)$ using $k=k_{t}$ with random Z2 and wall sources on a $24^{3} \times 64$ lattice.

Our analysis establishes the source operator dependence is well under control.

\section{Sink operator dependence}

The sink smearing of the pion operator in $C_{\pi \pi}(t)$ produces an extra overall factor. It can not be removed by the same ratio as that in the source operator case. Using a smearing function of the pion $f(x)$, the sink smeared BS wave function $\bar{\phi}(\mathbf{x} ; k)$ is obtained by

$$
\bar{\phi}(\mathbf{x} ; k)=\int d^{3} y f(|\mathbf{x}-\mathbf{y}|) \phi(\mathbf{y} ; k),
$$

where only one of the pion operators is smeared. $\bar{\phi}(\mathbf{x} ; k) / \bar{\phi}\left(\mathbf{x}_{\text {ref }} ; k\right)$ is not the same as $\phi(\mathbf{x} ; k) / \phi\left(\mathbf{x}_{\text {ref }} ; k\right)$ in contrast to the source operator case [8].

Nevertheless, the extra sink smearing factor can be analytically removed [24]. The sink smeared scattering amplitude $\bar{H}_{L}(p ; k)$ defined by $\bar{\phi}(\mathbf{x} ; k)$ relates to $H_{L}(p ; k)$ such that

$$
\begin{aligned}
\bar{H}_{L}(p ; k) & =-\int d^{3} x\left(\Delta+k^{2}\right) \bar{\phi}(\mathbf{x} ; k) e^{-i \mathbf{p} \cdot \mathbf{x}} \\
& =C_{f}(p) H_{L}(p ; k),
\end{aligned}
$$

where

$$
C_{f}(p)=\int d^{3} x f(x) e^{-i \mathbf{p} \cdot \mathbf{x}} .
$$

The local operator corresponds to $f(x)=\delta^{(3)}(x)$ with $C_{f}(p)=1$ in all $p$. Once $f(x)$ is given, $C_{f}(p)$ can be analytically calculated and removed from $\bar{H}_{L}(p ; k)$.

We numerically check the sink smearing independence of our result. $\bar{\phi}(\mathbf{x} ; k)$ and $\bar{H}_{L}(p ; k)$ are calculated by replacing the integration and $e^{-i \mathbf{p} \cdot \mathbf{x}}$ in Eqs. (A3)-(A5) to the summation and $j_{0}(p x)$. We employ an exponential sink smearing function $f(x)=e^{-A x}$ with a constant $A$. We confirm the values of $\bar{H}_{L}(p ; k) / C_{f}(p)$ with different $f(x)$ are consistent, unless $f(x)$ is too broad comparing to 
$L / 2-R$. A broad sink smearing function is found to lift up $h(\mathbf{x} ; k)$ in $|\mathbf{x}|>R$, which violates the sufficient condition Eq. (10).

\section{APPENDIX B: FORMULATION IN THE MOMENTUM SPACE}

Formulation of $H(p ; k)$ using the BS wave function in momentum space is summarized. We first explain the formulation in the infinite volume and continuum theory, and then present its lattice version.

The main difference from those in explained in Sec. II is appearance of the surface term. Only the surface term contributes to the on-shell scattering amplitude.

\section{Infinite volume}

The scattering amplitude with the infinite volume $H(p ; k)$ in the continuum theory is given by

$$
H(p ; k)=-\int_{-\infty}^{\infty} d^{3} x h_{\infty}(\mathbf{x} ; k) e^{-i \mathbf{p} \cdot \mathbf{x}} .
$$

The reduced wave function $h_{\infty}(\mathbf{x} ; k)$ is defined using the BS wave function in the infinite volume $\phi_{\infty}(\mathbf{x} ; k)$,

$$
h_{\infty}(\mathbf{x} ; k)=\left(\Delta+k^{2}\right) \phi_{\infty}(\mathbf{x} ; k),
$$

where $\Delta$ is the Laplacian. Substituting Eq. (B2) to Eq. (B1) with the partial integration yields

$$
H(p ; k)=\left(p^{2}-k^{2}\right) \tilde{\phi}_{\infty}(\mathbf{p} ; k) .
$$

$\tilde{\phi}_{\infty}(\mathbf{p} ; k)$ is the BS wave function in the momentum space,

$$
\tilde{\phi}_{\infty}(\mathbf{p} ; k)=\int_{-\infty}^{\infty} d^{3} x \phi_{\infty}(\mathbf{x} ; k) e^{-i \mathbf{p} \cdot \mathbf{x}} .
$$

Equation (B3) could be regarded as the LSZ reduction formula in the relative coordinate. It is constructed by the Fourier transformation of the BS wave function with a momentum factor. It corresponds to the LSZ reduction formula of Eq. (1). At on-shell, both formulas give the same on-shell scattering amplitude. In the zero momentum limit, Eq. (B3) leads to the scattering length $a_{0}$, as in Ref. [10]. Equation (B3) is not suitable, however, for the lattice calculation on a finite volume. $\phi_{\infty}(\mathbf{x} ; k)$ at all $\mathbf{x}$ in the infinite volume is demanded.

In the following, we consider a formulation with the finite integration range [9]. If $h_{\infty}(\mathbf{x} ; k)=0$ outside the interaction range $R$, then the integration range of $H(p ; k)$ can be changed from $\infty$ to $R$,

$$
\begin{aligned}
H(p ; k) & =-\int_{-\infty}^{\infty} d^{3} x h_{\infty}(\mathbf{x} ; k) e^{-i \mathbf{p} \cdot \mathbf{x}}, \\
& =-\int_{-R}^{R} d^{3} x h_{\infty}(\mathbf{x} ; k) e^{-i \mathbf{p} \cdot \mathbf{x}} .
\end{aligned}
$$

The partial integration gives

$$
\begin{aligned}
H(p ; k)= & \left(p^{2}-k^{2}\right) \int_{-R}^{R} d^{3} x \phi_{\infty}(\mathbf{x} ; k) e^{-i \mathbf{p} \cdot \mathbf{x}} \\
& -\sum_{i=1}^{3} \int_{-R}^{R} d^{2} x\left[\partial_{i} \phi_{\infty}(\mathbf{x} ; k)+i p_{i} \phi_{\infty}(\mathbf{x} ; k)\right]_{x_{i}=R},
\end{aligned}
$$

where the second term is the surface term. At on-shell $p=k$, the first term vanishes. $H(p ; k)$ is expressed by the surface term only.

In the spherical coordinate, $H(p ; k)$ can be simplified.

$$
\begin{aligned}
H(p ; k)= & 4 \pi\left(p^{2}-k^{2}\right) \int_{0}^{R} d x x^{2} \phi_{\infty}(\mathbf{x} ; k) j_{0}(p x) \\
& -\frac{4 \pi}{p}\left\{\left.R \sin (p R) \frac{\partial \phi_{\infty}(\mathbf{x} ; k)}{\partial x}\right|_{x=R}\right. \\
& \left.-(p R \cos (p R)-\sin (p R)) \phi_{\infty}(R ; k)\right\} .
\end{aligned}
$$

At on-shell, the expression of $H(k ; k)$ in Eq. (2) is reproduced by substituting the following $l=0 \phi_{\infty}(\mathbf{x} ; k)$ in $x>R$ to the surface term,

$$
\phi_{\infty}(\mathbf{x} ; k)=e^{i \delta(k)} \frac{\sin (k x+\delta(k))}{k x} .
$$

\section{Finite volume}

The lattice version of Eq. (B1) is

$$
H_{L}(p ; k)=-\sum_{i=1}^{3} \sum_{x_{i}=-L_{\min }}^{L_{\max }} C_{k} h(\mathbf{x} ; k) e^{-i \mathbf{p} \cdot \mathbf{x}},
$$

where $C_{k}$ is an overall constant in Eq. (11). $h(\mathbf{x} ; k)$ is the reduced wave function on the lattice. A choice of $L_{\max }=$ $L / 2$ and $L_{\min }=L / 2-1$ corresponds to the summation over the entire spatial volume with its extent $L . L_{\max }$ and $L_{\min }$ can be decreased, as long as $L_{\max }, L_{\min }>R$ is satisfied.

The partial integration on the lattice leads to

$$
\begin{aligned}
H_{L}(p ; k)= & \left(\tilde{p}^{2}-k^{2}\right) \sum_{i=1}^{3} \sum_{x_{i}=-L_{\min }}^{L_{\max }} C_{k} \phi(\mathbf{x} ; k) e^{-i \mathbf{p} \cdot \mathbf{x}} \\
& +\operatorname{surf}(p ; k),
\end{aligned}
$$

where

$$
\tilde{p}_{i}=\frac{2}{a} \sin \frac{a p_{i}}{2} .
$$

The surface term on the lattice $\operatorname{surf}(p ; k)$ is given by 


$$
\begin{aligned}
\operatorname{surf}(p ; k)= & -C_{k} \sum_{i=1}^{3} \sum_{x_{j, k}=-L_{\min }}^{L_{\max }}\left(e^{-i \mathbf{p} \cdot \mathbf{X}\left(L_{\max }\right)} \phi\left(\mathbf{X}\left(L_{\max }+1\right) ; k\right)\right. \\
& -e^{-i \mathbf{p} \cdot \mathbf{X}\left(L_{\max }+1\right)} \phi\left(\mathbf{X}\left(L_{\max }\right) ; k\right) \\
& -e^{-i \mathbf{p} \cdot \mathbf{X}\left(-L_{\min }-1\right)} \phi\left(\mathbf{X}\left(-L_{\min }\right) ; k\right) \\
& \left.+e^{-i \mathbf{p} \cdot \mathbf{X}\left(-L_{\min }\right)} \phi\left(\mathbf{X}\left(-L_{\min }-1\right) ; k\right)\right), \quad(\mathrm{B} 13)
\end{aligned}
$$

where $\mathbf{X}(a)=\mathbf{x}$ except for $X_{i}(a)=a$. The surface term is not zero, in general. If $L_{\max }=L / 2$ and $L_{\min }=L / 2-1$ are chosen and $p_{i} L_{\max }=n_{i} \pi, n_{i} \in \mathbf{Z}$ is satisfied under the periodic boundary condition, the surface term becomes zero.

In the case of the S-wave scattering on the lattice, $H_{L}(p ; k)$ becomes

$$
\begin{aligned}
H_{L}(p ; k)= & -k^{2} \sum_{i=1}^{3} \sum_{x_{i}=-L_{\min }}^{L_{\max }} C_{k} \phi(\mathbf{x} ; k) j_{0}(p x) \\
& -\sum_{i=1}^{3} \sum_{x_{i}=-L_{\min }}^{L_{\max }} C_{k} \phi(\mathbf{x} ; k) \Delta j_{0}(p x) \\
& +\operatorname{surf}(p ; k),
\end{aligned}
$$

where $\Delta$ is the symmetric lattice Laplacian defined in Eq. (9). The surface term in this case is given by

$$
\begin{aligned}
\operatorname{surf}(p ; k)= & -C_{k} \sum_{i=1}^{3} \sum_{x_{j, k}=-L_{\min }}^{L_{\max }}\left(j_{0}\left(p X\left(L_{\max }\right)\right) \phi\left(\mathbf{X}\left(L_{\max }+1\right) ; k\right)\right. \\
& -j_{0}\left(p X\left(L_{\max }+1\right)\right) \phi\left(\mathbf{X}\left(L_{\max }\right) ; k\right) \\
& -j_{0}\left(p X\left(-L_{\min }-1\right)\right) \phi\left(\mathbf{X}\left(-L_{\min }\right) ; k\right) \\
& \left.+j_{0}\left(p X\left(-L_{\min }\right)\right) \phi\left(\mathbf{X}\left(-L_{\min }-1\right) ; k\right)\right), \quad(\mathrm{B} 15)
\end{aligned}
$$

where $X(a)=|\mathbf{X}(a)|$. If $L_{\max }=L / 2$ and $L_{\min }=L / 2-1$ are chosen, the periodicity and isotropy of $\phi(\mathbf{x} ; k)$ leads to a simpler form of $\operatorname{surf}(p ; k)$,

$$
\begin{aligned}
\operatorname{surf}(p ; k)= & -3 C_{k} \sum_{x_{1,2}=-L_{\min }}^{L_{\max }}\left[j_{0}\left(p X^{\prime}\left(L_{\min }\right)\right)\right. \\
& \left.-j_{0}\left(p X^{\prime}\left(L_{\max }+1\right)\right)\right] \times \phi\left(\mathbf{X}^{\prime}\left(L_{\max }\right) ; k\right),
\end{aligned}
$$

where $X_{1,2}^{\prime}(a)=x_{1,2}$ and $X_{3}^{\prime}(a)=a$.

\section{APPENDIX C: $t$ INDEPENDENCE OF $\boldsymbol{H}_{L}(\boldsymbol{k} ; \boldsymbol{k}) /\left(\boldsymbol{C}_{\boldsymbol{k}} \boldsymbol{\phi}\left(\mathbf{x}_{\mathrm{ref}} ; \boldsymbol{k}\right)\right)$}

The $t$ dependence of $H_{L}(k ; k) /\left(C_{k} \phi\left(\mathbf{x}_{\text {ref }} ; k\right)\right)$ in Fig. 7 is discussed under several assumptions.
We define $H_{L}(t, k ; k)$ to study the scattering amplitude $H_{L}(k ; k)$ at each $t$,

$$
H_{L}(t, k ; k)=-\sum_{\mathbf{x}}\left(\Delta+k^{2}\right) C_{\pi \pi}(\mathbf{x}, t) j_{0}(k x) .
$$

We evaluate a ratio of $H_{L}(t, k ; k) / C_{\pi \pi}(\mathbf{x}, t)$. It can be split into the ground state and the excited state parts,

$$
\frac{H_{L}(t, k ; k)}{C_{\pi \pi}\left(\mathbf{x}_{\mathrm{ref}}, t\right)}=\frac{H_{L}(k ; k)}{C_{k} \phi\left(\mathbf{x}_{\mathrm{ref}} ; k\right)} \frac{1+\delta H_{L}(t, k ; k)}{1+\delta C_{\pi \pi}\left(\mathbf{x}_{\mathrm{ref}}, t\right)},
$$

where the excited state contributions $\delta H_{L}(t, k ; k)$ and $\delta C_{\pi \pi}\left(\mathbf{x}_{\text {ref }}, t\right)$ for the numerator and denominator, respectively. Figure 7 illustrates $H_{L}(t, k ; k) / C_{\pi \pi}\left(\mathbf{x}_{\text {ref }}, t\right)$ is almost flat against $t$. On the other hand, Fig. 8 clearly reveals nonnegligible contributions of $\delta H_{L}(t, k ; k)$ and $\delta C_{\pi \pi}\left(\mathbf{x}_{\text {ref }}, t\right)$ in the small $t$ region. It suggests a possibility of cancellation between the numerator and the denominator,

$$
\delta H_{L}(t, k ; k) \sim \delta C_{\pi \pi}\left(\mathbf{x}_{\text {ref }}, t\right) .
$$

This is a sufficient condition of flat $t$ dependence of $H_{L}(t, k ; k) / C_{\pi \pi}\left(\mathbf{x}_{\text {ref }}, t\right)$.

We demonstrate the sufficient condition is realized, if $C_{\pi \pi}\left(\mathbf{x}_{\mathrm{ref}}, t\right)$ is dominated by scattering states with almost zero momentum. In the small $t$ region, $C_{\pi \pi}(\mathbf{x}, t)$ includes large contributions from not only the ground state of two pions, but also scattering states with the first radial excited state of $\pi$, denoting $\pi^{\prime}$. We restrict our consideration below the energy of $\pi^{\prime} \pi^{\prime} \rightarrow \pi^{\prime} \pi^{\prime}$ scattering, neglecting inelasticities. Then, $C_{\pi \pi}(\mathbf{x}, t)$ is expressed as

$$
\begin{aligned}
C_{\pi \pi}(\mathbf{x}, t)= & \sum_{q} A_{q}(t) \phi(\mathbf{x} ; q)+\sum_{q^{\prime}} A_{q^{\prime}}^{\prime}(t) \phi^{\prime}\left(\mathbf{x} ; q^{\prime}\right) \\
& +\sum_{q^{\prime \prime}} A_{q^{\prime \prime}}^{\prime \prime}(t) \phi^{\prime \prime}\left(\mathbf{x} ; q^{\prime \prime}\right),
\end{aligned}
$$

where $A_{q}(t)=C_{q} e^{-E_{q^{\prime}} t}, A_{q^{\prime}}^{\prime}(t)=C_{q^{\prime}}^{\prime} e^{-E_{q^{\prime}}^{\prime}}$, and $A_{q^{\prime \prime}}^{\prime \prime}(t)=$ $C_{q^{\prime \prime}}^{\prime \prime} e^{-E_{q^{\prime \prime}}^{\prime \prime} t}$ where $C_{q}, C_{q^{\prime}}^{\prime}$, and $C_{q^{\prime \prime}}^{\prime \prime}$ are the overall constants for each contribution, and

$$
\begin{aligned}
& E_{q}=2 \sqrt{m_{\pi}^{2}+q^{2}}, \\
& E_{q}^{\prime}=\sqrt{m_{\pi}^{2}+q^{2}}+\sqrt{m_{\pi^{\prime}}^{2}+q^{2}}, \\
& E_{q}^{\prime \prime}=2 \sqrt{m_{\pi^{\prime}}^{2}+q^{2}} .
\end{aligned}
$$

The terms with the prime (') and double prime (") correspond to contributions of scatterings for $\pi \pi^{\prime} \rightarrow \pi \pi^{\prime}$ and $\pi^{\prime} \pi^{\prime} \rightarrow \pi^{\prime} \pi^{\prime}$. Substituting Eq. (C4) to Eq. (C1) provides 


$$
\begin{aligned}
H_{L}(t, k ; k)= & \sum_{q} A_{q}(t) \operatorname{surf}(k ; q)+\sum_{q^{\prime}} A_{q^{\prime}}^{\prime}(t) \operatorname{surf}^{\prime}\left(k ; q^{\prime}\right) \\
& +\sum_{q^{\prime \prime}} A_{q^{\prime \prime}}^{\prime \prime}(t) \operatorname{surf}^{\prime \prime}\left(k ; q^{\prime \prime}\right),
\end{aligned}
$$

where we use $\operatorname{surf}(p ; k)$ in Eq. (B16) in conjunction with $\Delta j_{0}(k x)=-k^{2} j_{0}(k x)$, ignoring the lattice artifact for simplicity,

$$
-\sum_{\mathbf{x}}\left(\Delta+k^{2}\right) \phi(\mathbf{x} ; q) j_{0}(k x)=\operatorname{surf}(k ; q) .
$$

The excited state contributions $\delta H_{L}(t, k ; k)$ and $\delta C_{\pi \pi}\left(\mathbf{x}_{\mathrm{ref}}, t\right)$ for the numerator and denominator are expressed as

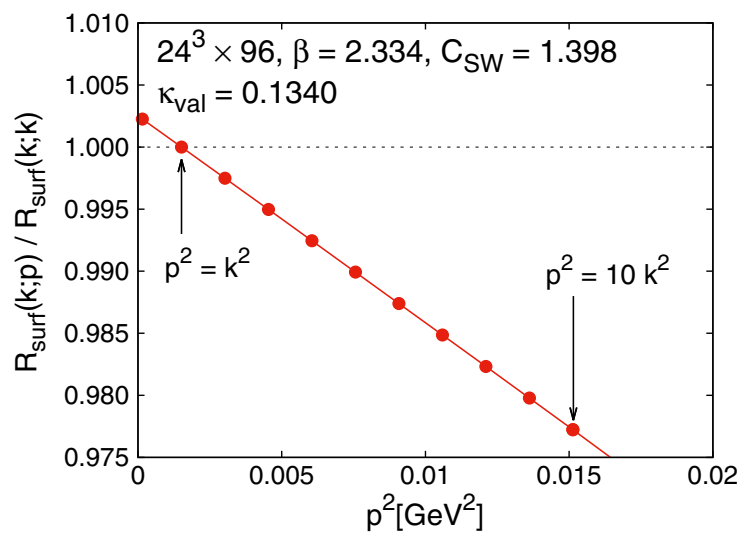

FIG. 15. $\quad p^{2}$ dependence of the ratio of $R_{\text {surf }}(k ; p) / R_{\text {surf }}(k ; k)$ with $\mathbf{x}_{\mathrm{ref}}=(12,7,2)$.

$$
\begin{aligned}
& \delta H_{L}(t, k ; k)=\frac{\sum_{q \neq k} A_{q}(t) \operatorname{surf}(k ; q)+\sum_{q^{\prime}} A_{q^{\prime}}^{\prime}(t) \operatorname{surf}{ }^{\prime}\left(k ; q^{\prime}\right)+\sum_{q^{\prime \prime}} A_{q^{\prime \prime}}^{\prime \prime}(t) \operatorname{surf}^{\prime \prime}\left(k ; q^{\prime \prime}\right)}{A_{k}(t) \operatorname{surf}(k ; k)}, \\
& \delta C_{\pi \pi}\left(\mathbf{x}_{\mathrm{ref}}, t\right)=\frac{\sum_{q \neq k} A_{q}(t) \phi\left(\mathbf{x}_{\mathrm{ref}} ; q\right)+\sum_{q^{\prime}} A_{q^{\prime}}^{\prime}(t) \phi^{\prime}\left(\mathbf{x}_{\mathrm{ref}} ; q^{\prime}\right)+\sum_{q^{\prime \prime}} A_{q^{\prime \prime}}^{\prime \prime}(t) \phi^{\prime \prime}\left(\mathbf{x}_{\mathrm{ref}} ; q^{\prime \prime}\right)}{A_{k}(t) \phi\left(\mathbf{x}_{\mathrm{ref}} ; k\right)} .
\end{aligned}
$$

The cancellation condition of Eq. (C3) implies the coefficients of each state contribution in $\delta H_{L}(t, k ; k)$ and $\delta C_{\pi \pi}\left(\mathbf{x}_{\text {ref }}, t\right)$ coincide. At some momentum $p$, the relation between the coefficients is

$$
\frac{\operatorname{surf}(k ; p)}{\operatorname{surf}(k ; k)} \sim \frac{\phi\left(\mathbf{x}_{\text {ref }} ; p\right)}{\phi\left(\mathbf{x}_{\text {ref }} ; k\right)} .
$$

$\phi\left(\mathbf{x}_{\mathrm{ref}} ; p\right)$ for $x_{\text {ref }}>R$ can be expressed by the solution of the Helmholtz equation $G(\mathbf{x} ; p)$ defined in Eq. (26). The surface terms are also evaluated by $G(\mathbf{x} ; p)$ through Eq. (B16), supposing the surface boundary lies outside the interaction range $R$. Substituting $G(\mathbf{x} ; p)$ for $\phi\left(\mathbf{x}_{\text {ref }} ; p\right)$ and $\operatorname{surf}(k ; p)$ in $p^{2} \sim k^{2}$ leads to

$$
\frac{\operatorname{surf}(k ; k)}{G\left(\mathbf{x}_{\text {ref }} ; k\right)} \sim \frac{\operatorname{surf}(k ; p)}{G\left(\mathbf{x}_{\text {ref }} ; p\right)} .
$$

Equation (C13) is numerically estimated. We define a ratio $R_{\text {surf }}(k ; p)$ for the estimation,

$$
R_{\text {surf }}(k ; p)=\frac{\operatorname{surf}(k ; p)}{G\left(\mathbf{x}_{\text {ref }} ; p\right)} .
$$

We investigate $p^{2}$ dependence of $R_{\text {surf }}(k ; p)$ in the range of $0.1 k^{2} \leq p^{2} \leq 10 k^{2}$, supposing unmeasured $q_{0}^{\prime}$ and $q_{0}^{\prime \prime}$ are in this range. The result is plotted in Fig. 15. Difference of $R_{\text {surf }}(k ; p)$ is less than $3 \%$ even at $p^{2}=10 k^{2}$. Our data support validity of Eq. (C12).

In summary, the excited states contamination in the ratio of $H_{L}(k ; k) /\left(C_{k} \phi\left(\mathbf{x}_{\text {ref }} ; k\right)\right)$ is understood under the following conditions.

(i) Energy is below $\pi^{\prime} \pi^{\prime} \rightarrow \pi^{\prime} \pi^{\prime}$ scattering with no inelasticities.

(ii) Contribution from higher momentum states is small, due to our choice of the source operator.

(iii) $q_{0}^{\prime}$ and $q_{0}^{\prime \prime}$ are assumed to be in the range of $0.1 k^{2} \leq p^{2} \leq 10 k^{2}$.

Then, contamination between $H_{L}(t, k ; k)$ and $C_{\pi \pi}\left(\mathbf{x}_{\text {ref }}, t\right)$ can be explained. For further analysis, we need the variational method to distinguish the excited states. 
[1] R. A. Briceno, J. J. Dudek, and R. D. Young, Rev. Mod. Phys. 90, 025001 (2018).

[2] R. A. Briceño, EPJ Web Conf. 175, 01016 (2018).

[3] M. Lüscher, Commun. Math. Phys. 105, 153 (1986).

[4] M. Lüscher, Nucl. Phys. B354, 531 (1991).

[5] C. J. D. Lin, G. Martinelli, C. T. Sachrajda, and M. Testa, Nucl. Phys. B619, 467 (2001).

[6] S. Aoki, M. Fukugita, K.-I. Ishikawa, N. Ishizuka, Y. Iwasaki, T. Kaneko, Y. Kuramashi, M. Okawa, A. Ukawa, T. Yamazaki, and T. Yoshié (CP-PACS Collaboration), Phys. Rev. D 71, 094504 (2005).

[7] S. Aoki, T. Hatsuda, and N. Ishii, Prog. Theor. Phys. 123, 89 (2010).

[8] T. Yamazaki and Y. Kuramashi, Phys. Rev. D 96, 114511 (2017).

[9] Y. Namekawa and T. Yamazaki, Phys. Rev. D 98, 011501 (2018).

[10] J. Carbonell and V. A. Karmanov, Phys. Lett. B 754, 270 (2016).

[11] J. J. Dudek, R. G. Edwards, and C. E. Thomas, Phys. Rev. D 86, 034031 (2012).

[12] L. Lellouch and M. Luscher, Commun. Math. Phys. 219, 31 (2001).

[13] A. A. Khan, S. Aoki, G. Boyd, R. Burkhalter, S. Ejiri, M. Fukugita, S. Hashimoto, N. Ishizuka, Y. Iwasaki, K. Kanaya, T. Kaneko, Y. Kuramashi, T. Manke, K. Nagai, M. Okawa, H. P. Shanahan, A. Ukawa, and T. Yoshié
(CP-PACS Collaboration), Phys. Rev. D 65, 054505 (2002); 67, 059901(E) (2003).

[14] Y. Iwasaki, arXiv:1111.7054; Nucl. Phys. B258, 141 (1985).

[15] B. Sheikholeslami and R. Wohlert, Nucl. Phys. B259, 572 (1985).

[16] P. A. Boyle, A. Juttner, C. Kelly, and R. D. Kenway, J. High Energy Phys. 08 (2008) 086.

[17] J. Gasser and H. Leutwyler, Ann. Phys. (N.Y.) 158, 142 (1984).

[18] G. Colangelo, J. Gasser, and H. Leutwyler, Nucl. Phys. B603, 125 (2001).

[19] S. R. Beane, E. Chang, W. Detmold, H. W. Lin, T. C. Luu, K. Orginos, A. Parreno, M. J. Savage, A. Torok, and A. Walker-Loud (NPLQCD Collaboration), Phys. Rev. D 85, 034505 (2012).

[20] J. Bijnens, G. Colangelo, G. Ecker, J. Gasser, and M. E. Sainio, Nucl. Phys. B508, 263 (1997); B517, 639(E) (1998).

[21] S. Ueda, S. Aoki, T. Aoyama, K. Kanaya, H. Matsufuru, S. Motoki, Y. Namekawa, H. Nemura, Y. Taniguchi, and N. Ukita, J. Phys. Conf. Ser. 523, 012046 (2014).

[22] Y. Kuramashi, M. Fukugita, H. Mino, M. Okawa, and A. Ukawa, Phys. Rev. Lett. 71, 2387 (1993).

[23] M. Fukugita, Y. Kuramashi, M. Okawa, H. Mino, and A. Ukawa, Phys. Rev. D 52, 3003 (1995).

[24] D. Kawai, S. Aoki, T. Doi, Y. Ikeda, T. Inoue, T. Iritani, N. Ishii, T. Miyamoto, H. Nemura, and K. Sasaki (HAL QCD Collaboration), Prog. Theor. Exp. Phys. 2018, 043 B04 (2018). 